\title{
Socio-inspired ICT
}

\section{Towards a socially grounded society-ICT symbiosis}

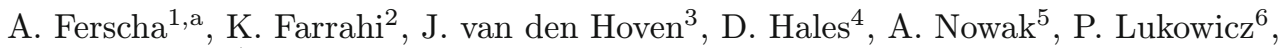
and D. Helbing ${ }^{7}$

1 University of Linz (JKU), Inst. f. Pervasive Computing, Altenberger Strasse 69, 4040 Linz, Austria

${ }^{2}$ University of Linz (JKU), Inst. f. Pervasive Computing, Altenberger Strasse 69, 4040 Linz, Austria

3 Philosophy Section, Delft University of Technology, Jaffalaan 5, PO Box 5015, 2600 GA Delft, The Netherlands

4 The Open University, London, UK

5 Department of Psychology, University of Warsaw, 00-183 Warsaw, Poland Stawki 5/7, Poland

6 DFKI, Trippstadter Strae 122, D-67663 Kaiserslautern, Germany

7 ETH Zürich, Clausiusstrasse 50, 8092 Zürich, Switzerland

Received in final form 9 October 2012

Published online 5 December 2012

\begin{abstract}
Modern ICT (Information and Communication Technology) has developed a vision where the "computer" is no longer associated with the concept of a single device or a network of devices, but rather the entirety of situated services originating in a digital world, which are perceived through the physical world. It is observed that services with explicit user input and output are becoming to be replaced by a computing landscape sensing the physical world via a huge variety of sensors, and controlling it via a plethora of actuators. The nature and appearance of computing devices is changing to be hidden in the fabric of everyday life, invisibly networked, and omnipresent, with applications greatly being based on the notions of context and knowledge. Interaction with such globe spanning, modern ICT systems will presumably be more implicit, at the periphery of human attention, rather than explicit, i.e. at the focus of human attention.

Socio-inspired ICT assumes that future, globe scale ICT systems should be viewed as social systems. Such a view challenges research to identify and formalize the principles of interaction and adaptation in social systems, so as to be able to ground future ICT systems on those principles. This position paper therefore is concerned with the intersection of social behaviour and modern ICT, creating or recreating social conventions and social contexts through the use of pervasive, globe-spanning, omnipresent and participative ICT.
\end{abstract}

\footnotetext{
${ }^{a}$ e-mail: ferscha@pervasive.jku.at
} 


\section{The rise of aware ICT}

Modern ICT, building on the ever progressing miniaturization of technology (processing, storage, communication) as well as at the ever growing globe spanning networks, has postulated to invisibly integrate technology into everyday objects like tools, appliances, objects of everyday use, and environments like offices, homes and cars in such a way, that these objects turn into "smart things" or "smart environments". Built with networked embedded systems technology, such "smart" things and environments have become increasingly interconnected, diverse and heterogeneous, and together with IP networking technology have created a whole new generation of ICT as we see it today (e.g. the "Internet-of-Things", "Smart Buildings, Cars, Cities", "Smart Grids", even the "Smart Planet"). Only networking and communication capabilities, however, cannot make things and environments appear "smart", unless coping with the challenge of an operative, and semantically meaningful interplay among each other.

One approach to address the challenge of "smart" ICT has been to design and implement systems able to manage themselves in a more or less autonomous way, with little or no human interaction. While self-management stands for the ability of single smart thing to describe itself, to select and use adequate sensors to capture information describing its context, self-organizing stands for the ability of a group of possibly heterogeneous peers to establish a spontaneous network based on interest, purpose or goal, and to negotiating and fulfilling a group goal. Self-management relates to an individual smart thing, and concerns adaptation to changing individual goals and conditions at runtime, while self-organization relates to whole ensembles of smart things, and concerns adaptation in order to meet group goals.

A prerequisite for self-management, even more so for self-organization is the ability to autonomously perceive, recognize, and even anticipate phenomena and their consequences, i.e. being "aware". Early signs "aware ICT" have been observed by contributions from Pervasive and Ubiquitous Computing over the past two decades, starting with systems being aware about the physical situation they are operated in ("context aware ICT")[134], and later on with systems being aware about the user and his activities ("activity aware ICT") [120,132] (see Fig. 1). More recent trends tend to make ICT aware about social ("socially aware ICT") [96,118], emotional ("emotion aware ICT") [9] and even cognitive aspects (e.g., "attention aware ICT") [47]. We look at this evolution in more detail.

\subsection{From context aware ICT to socio-technical fabric}

Quoting from Weiser's (1991) vision "The most profound technologies are those that disappear. They weave themselves into the fabric of every day life, until they are indistinguishable from it" [151] conveys the most common understanding of the origins of a computer science research branch today known as Pervasive and Ubiquitous Computing (PUC). Weiser's seminal vision was pathbreaking, and still represents the corner stone for what might be referred to as a first generation of research, aiming towards embedded, hidden, invisible, but networked ICT systems. This first generation definitely gained from the technological progress momentum (miniaturization of electronics, gate packaging), and was driven by the upcoming availability of technology to connect literally everything to everything (Connectedness, Late Nineties), like wireless communication standards and the exponentially growing internet. Networks of systems emerged, forming communication clouds of miniaturized, cheap, fast, powerful, wirelessly connected, "always on" systems, enabled by the massive availability of miniaturized computing, storage, communication, and embedded systems technologies. Special purpose computing and information appliances, ready to spontaneously 


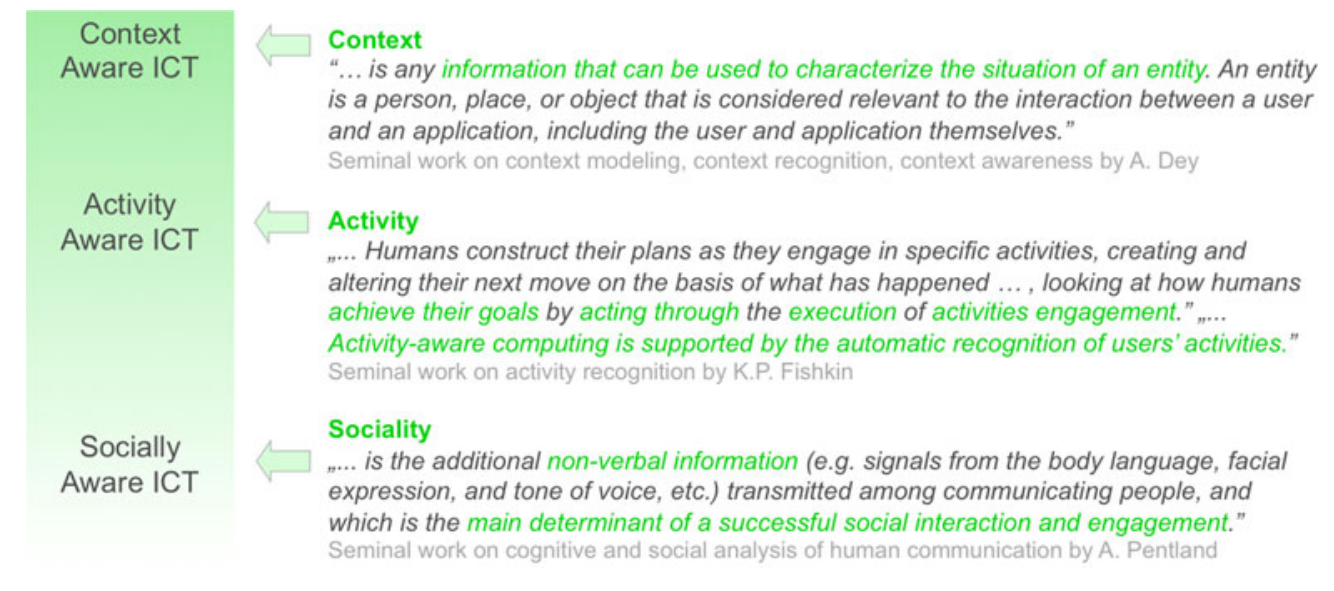

Fig. 1. The Evolution of Aware ICT Systems.

communicate with one another, sensor-actuator systems to invert the roles of interaction from human to machine (implicit interaction), and organism like capabilities (self-configuration, self-healing, self-optimizing, self-protecting) characterize this generation.

The second generation PUC research inherited from the then upcoming sensor based recognition systems, as well as knowledge representation and processing technologies (Awareness, around the turn of the century), where research issues like e.g. context and situation awareness, self-awareness, future-awareness or resourceawareness reshaped the understanding of pervasive computing. Autonomy and adaptation in this generation was reframed to be based on knowledge, extracted from low level sensor data captured in a particular situation or over long periods of time (The respective "epoch" of research on "context aware" systems was stimulated by Schillit, Adams and Want [134], and fertilized by the PhD work of Anind Dey [31], redefining the term "context" as: "... any information that can be used to characterize the situation of an entity. An entity is a person, place, or object that is considered relevant to the interaction between a user and an application, including the user and application themselves."). One result out of this course of research are autonomic systems [72], and later autonomic elements, able to capture context, to build up, represent and carry knowledge, to self-describe, -manage, and-organize with respect to the environment, and to exhibit behaviour grounded on "knowledge based" monitoring, analysing, planning and executing were proposed, shaping ecologies of ICT systems, built from collective autonomic elements interacting in spontaneous spatial/temporal contexts, based on proximity, priority, privileges, capabilities, interests, offerings, environmental conditions, etc.

Finally, a third generation of PUC is approaching, building upon connectedness and awareness, and attempting to exploit the (ontological) semantics of systems, services and interactions (i.e. giving meaning to situations and actions). Such systems are often referred to as highly complex, orchestrated, cooperative and coordinated "Ensembles of Digital Artefacts" (FP7 FET). An essential aspect of such an ensemble is its spontaneous configuration towards a complex system, i.e. a "... dynamic network of many agents (which may represent cells, species, individuals, nations) acting in parallel, constantly acting and reacting to what the other agents are doing where the control tends to be highly dispersed and decentralized, and if there is to be any coherent behavior in the system, it has to arise from competition and cooperation 
among the agents, so that the overall behavior of the system is the result of a huge number of decisions made every moment by many individual agents" [19].

Ensembles of digital artefacts as compounds of huge numbers of possibly heterogeneous entities constitute a future generation of systems to which we refer as SocioTechnical Fabric [49], weaving social and technological phenomena into the 'fabric of technology-rich societies'. Indications of evidence for such large scale, complex, technology rich societal settings are facts like $10^{12}-10^{13}$ "things" or "goods" being traded in (electronic) markets today, $10^{9}$ personal computer nodes and $10^{9}$ mobile phones on the internet, $10^{8}$ cars or $10^{8}$ digital cameras with sophisticated embedded electronics - even for internet access on the go, etc. Today's megacities approach sizes of $10^{7}$ citizens. Already today some $10^{8}$ users are registered on Facebook, $10^{8}$ videos have been uploaded to YouTube, like $10^{7}$ music titles haven been labeled on last.fm, etc. Next generation research directions are thus going away from single user, or small user group as addressed in the first two generations, heading more towards complex socio-technical systems, i.e. large scale to very large scale deployments of PUC and the respective concerns on a societal level.

\subsection{Key future ICT research challenges identified by the scientific community}

To better understand the trends and impacts of future ICT systems, as well as the research challenges posed by them, we have conducted a large scale solicitation initiative to pave future generation ICT research roadmaps ${ }^{1}$ The voices raised by active researchers in the field can be clustered according to the following research challenges for future generation PUC [48].

Autonomous adaptation. The first category of challenges articulated by the scientific community addresses on systems related research concerning the ability of a system to adapt to situation changes based on an autonomous recognition and assessment of the situation, and to "facilitate the survival of the system". Parallels can be identified to the ongoing self-* systems research, but issues are raised that reach far into foreign domains like neurology, immunology (e.g. systems developing their own internal self-image to guide interaction with the externa, E. Hart), or environmental research (e.g. systems self-optimizing their configuration with respect to. to environmental constraints like carbon footprint or global energy, D. Moriandi). The study of symbiotic multi-body organisms and systems with homeostatic abilities (e.g. danger perception or self/non-self discrimination, J. Timmis) is proposed, much like the relief of mobility and spatial coverage constraints in wireless sensor networks (e.g. collaborative "cloud sensing" with the robotic flying sensor network, P. Zhang).

Adaptive pervasive ensembles. Heterogeneous multipart systems provisioning services as an orchestrated service ensemble are challenging the community in several concerns. First, the integrative aspect of service components on a hardware and middleware layer and how they adapt to achieve service stability (K. Herrmann), second configuration aspect on the wireless communication topology layer (e.g. nanoscale communications and nanonetworks, O. Baris Akan), but also at the layer of orchestrated human-technology interaction at societal scale (socially interactive computing,

\footnotetext{
1 The FP7 FET proactive project PANORAMA (FET proactive/Goal 8.3: Pervasive Adaptation) picked up on the challenge of identifying the new trails of Pervasive Computing research, involving some 240 of the most distinguished researchers in the field in a solicitation process that lasted for about three years. The result of this process is manifested in the Pervasive Adaptation Research Agenda Book (www.perada.eu/research-agenda), an evolving document where the scientific community can contribute to and download the latest version in a ready to print layouted format.
} 
P. Lukowicz, D. Helbing and St. Bishop). This latter research challenge statement (backed by whole FuturICT project) is even addressing globe-spanning, complex, dynamic systems, where "adaptation could range from reshuffling of resources (e.g. information sources, bandwidth, distributed computing resources) to enable a better monitoring and management of emerging crisis situations, over the mediation of interactions in and between communities, up to emergency 'slow down and ask human' mechanisms, preventing the system from accelerating crises and systemic failures" (P. Lukowicz). Symptomatically, like many of the recently evolving research themes that build on todays capability to collect and analyze data at a scale that may reveal patterns of behavior of whole societies or even mankind (e.g. internet traffic, mobile telephony, automotive mobility, energy consumption, etc.), this category attempts for a sustainable, reliable, stable, trustworthy and inclusive ICT with human society, rather than an individual user as the target.

Emergence and evolvability. Understanding the principles of growing ICT systems according to phenomena like emergence (i.e. the way how complex systems and structures arise from the combination and multiplicity of very simple components or interactions), or evolvability (the idea of evolvable systems originated from early research in cybernetics, where evolve-ability is known as "the ability of a population to produce variants fitter than any yet existing") is considered a research challenge to cope with the seamless integration of future technologies with already existing ICT infrastructures. Systems must have the ability to adapt to spontaneous, unforeseen and even frequently changing technological infrastructures, while preserving the capability of interfacing to established technologies. Changes in system design paradigms from constructive to evolvable ("It is natural that we ask ourselves if it is possible to start with a minimal architecture and let the system grow and develop by itself, as an answer to the environment demands and system's goals." E. Costa), or instructive ("by 'instructing' each tiny component from a network of components to increase a specific benefit", G. Persiona) and long-term "self-developmental" (S. Kernbach) is where scientists identify need for research. The challenge to harmonize -at least a temporal coexistence of- radical innovations with existing technology is made clear by the example of $4 \mathrm{D}$ images (O. Bimber): color encoding only spatial information of a scene in pixels of raster-displayed images (2D) could be enhanced to also encode angular information, e.g. individual color for each emitting direction of a pixel (4D). How would 4D light field photography/cameras and light field displays coexist with traditional 2D imaging as a ICT enabling technology?

Societies of artefacts. Conceivably, future ICT will be manifested by technology rich artefacts (like tools, appliances, objects of every day use), and environments (like work and home places, or sports and entertainment locations), cooperatively attempting service provision with society-like behavior. Going beyond their capability to localize and recognize other artefacts as well as humans and their intentions, societal artefacts will form up to "goal tribes", i.e. ensembles of possibly complementing competencies, to act in a sensitive, proactive, and responsive way according to the perceived and anticipated needs, habits, and emotions of the users. While the social ability of such artefacts is just the demanding prerequisite, the ability to form goal driven interest communities according to societal models is the potential approach to harness an ever increasing complexity of ICT. Coordinated goal oriented artifact communities ("engineered to form societies, interact and compete with other ecologies, collaborate with humans and develop their own methods of conception and social norms", A. Kameas) are supposed to be the "interface", via which humans will ultimately be served. Research on the conception of artifact societies may supposedly inherit from social and cognitive sciences, as it appears to be challenged to 
understand the "innovative intersection of norm-governed systems, voting algorithmics, game theory, opinion formation, belief revision, judgement aggregation, and social computational choice ..., as well as a formal characterization of socio-cognitive principles of trust, forgiveness, and affect" (J. Pitt).

Dependable pervasive systems. Particularly for ICT systems of very large deployment, non-functional and quality-of-service system properties become prevalent over the pure services. Beyond traditional dependable systems research (availability, reliability, fault tolerance, maintainability), normative, "self-regulating" system design approaches are requested (a normative system refers to any distributed interaction system whose behavior can be regulated by norms, e.g. norms to meet stability objectives or to sustain certain utility levels) to be investigated. Much like individual human behavior locally "controlled" by (social, ethical, etc.) norms yields rational societies, ICT artefacts could be organized as ensembles of "mission components capable of assimilating and acting upon local intelligence 'on the fly" (J. Pitt). Aside technological QoS criteria, more outreaching notions of dependability research issues are proposed, like e.g. "sustainable" ("in terms of cost, life-cycle management or energy efficiency", St. Haller), "socially meaningful" (P. Lukowicz), privacy preserving, or avoiding electronic waste and "recyclability" (V. Namboodiri).

Pervasive trust. Among the most prevalent, ubiquitously recognized, and meanwhile also socially pressing research agenda items relate to the concerns humans might have in using and trusting ICT. Well beyond the privacy and trust related research we see in the Pervasive/Ubiquitous Computing research community already today, go the claims for trust research in the context of information spread via the internet, in emergency scenarios (e.g. advice-taking from technology or strangers), disaster management (tsunamis, floodings, nuclear disasters, riots and civil commotion, etc., E. Mitleton-Kelly) or risk analysis and crises forecasting (D. Helbing). The proposed working definition of trust; "... willingness to rely on another party and to take action in circumstances where such action makes one vulnerable to the other party" (E. Mitleton-Kelly) already indicates its relation to risk (due to uncertainty), and vulnerability (due to the readiness to act). Inspired by recent results on the assessment of trust users have in information delivered to ICT in particular situations (e.g. vibro-tactile directional guidance in evacuation situations, www.socionical.eu) are upcoming questions on how trust builds up (as a cognitive process from intuition, belief, experience/knowledge and expectation), how to integrate trust building processes into ICT, how to cope with distrust or lost trust, etc. The community agrees, that serious research on Pervasive Trust cannot survive without a formalisation of the trust related cognitive capabilities and terms (experience, belief, expectation), and a foundational underpinning with empirical evidence on trust mechanisms.

Human-centric adaptation. The standard phrase to approach a small-talk conversation among ICT scientist is the question: "Is it that human needs, capabilities or constraints shape the design and emergence of PUC technology, or do humans adapt to technology designs once it is deployed?" Clearly, technology innovation and the processes of technology adoption by humans is self-referential. Among the many examples throughout the evolution of ICT which have made it to industrial mass products and/or commercial success, are smartphones (the need to voice communicate vs. the joy of playful media and service access), car navigation systems (the need for wayfinding vs. online traffic management) or the internet of things (the need for identity management vs. total surveillance). Opposed to a (yet vibrant) HCI research agenda, which usually assumes users and systems as objects, and their interaction as subject of investigation, researchers now try to explain adaptation based on how users "understand" technology, raising "mental models" about how PUC systems 
work to become subject of investigation. "Intelligibility" (coined as "helping users to form an accurate mental model about how to use an application", A. Dey) and the design of ICT systems that lifelong and "continuously evaluate the degree of satisfaction (or frustration) they elicit in users" based on explicit but also implicit feedback (A. Roggen), that allow users "... to ask why did the system take a particular action, why didn't it take a different specific action" (A. Dey), that analyse physiological dynamics of users (based on sensory input "such as heartbeat, brain waves, blood pressure, oxygen saturation, muscular activity, respiration, body temperature, etc.", J. Kantelhart) as a statistical time series, that "truly understand our mental and emotional situation and try to accommodate us" (J. Healy), or that trace and extract "life patterns" as indicators of lifestyles (M. Mamei) - all in order to better understand how users "understand" technology. Recent research has coined the term "scrutability" to underline the necessity for users to be always able to inspect the knowledge of the system about them, and also to help users to understand system failures and their causes. We could refer to intelligible system designs also as "respectful", i.e. respecting 'peoples' ability to judge for themselves and be assisted by machines where needed. Respect for peoples' desire for freedom of choice and be supported by automation and decision support where appropriate" (S. Spiekermann).

Socio-technical systems. A significant trend in next generation ICT research, already observed taking its first steps, are investigations along the boundaries where technology and social behavior interact. From the observation that success PUC technologies (smartphones, mobile internet, autonomous driver assistance systems, social networks, etc.) have radically transformed individual communication and social interaction, the scientific community claims for "new foundations for ... largescale Human-ICT organisms and their adaptive behaviours, also including lessons form applied psychology, sociology, and social anthropology, other than from systemic biology, ecology and complexity science." (F. Zambonelli). Moving research attention from PUC for individual users towards the interplay of a complex, globe spanning, dynamically changing ICT and societies of billions of users worldwide ("Socially Interactive Computing", P. Lukowicz) reveals the inadequacy of ceteris paribus analysis: at levels of such scale and complexity, behavioral phenomena cannot be explained by investigations of influence quantities in isolation. Integrative views and research methods have to be adopted to explain technology influenced social dynamics. The community is challenged to extend the notion of "context aware" towards "socially aware" ICT [118]. Remember, according to Dey, a system is called context aware if it makes use of potentially any information that describes its situation. In analogy, we would call a system socially aware, if it makes use of potentially any information that describes its social habitus, i.e. any information which can be inferred from all of its past and present social relations, social interactions and social states. As for today, only a small subset of information constituting social context can be captured via (technical) sensors (use patterns of social networking tools, communication and mobility patterns captured by mobile devices, social apps, calendar sharing, embedded geo tags, road pricing, financial transactions, etc.), or is not conveniently accessible (hosted by public registries, state authorities, service providers), but is available in principle, and has stimulated research domains like computational social science [88].

Quality of life. To support human beings towards a better life, the wellbeing of individuals, as well as the welfare of societies are the quintessential prospect of PUC. Taking all the technological capabilities and potentials, as well as the human desire for wellbeing ("... a good condition of existence characterized by health, happiness, and prosperity of individuals in relation to their inner and outer personal spheres", O. Mayora) together, several questions arise on how to reach a satisfactorily wellbeing 
state via ICT. While lead and motivated by such humanistic principles towards making the "world a better place", it appears that the scientific community, at least for the time being, has not yet found substantiated research questions. More are there expressions of desirable world states ("Green World", M. Ulieru) and appreciable life styles ("freedom", A. Schmidt, "stress-free society", M. Mamei), reflecting what i would call "realistic fiction", but awaiting more specificity, structure and method.

Some selected, yet indicative voices raised by the scientific community towards the next generation ICT research challenges deserve to be highlighted:

Intelligibility. "One particular usability aspect of interest is intelligibility, helping users to form an accurate mental model about how to use an application. This is important for allowing users to understand how the application works and to be able to predict what it will do in a future situation, and all of this will impact adoption and use." (Anind K. Dey)

Social and cultural knowledge. "Advanced data analysis tools will allow spotting trends, observing their movement, their causes, and triggers. This platform, will allow researchers to ...explore social and cultural knowledge. What do people believe? And how people act." (Adrian D. Cheok)

Life-long satisfaction. "We believe that the next frontier in pervasive smart assistance will be to devise systems capable of continuous - lifelong - co-adaptation to the user needs. ... However, towards what should be the system adapted to, and by what should adaptation be driven? We believe there is no better way than to be guided by the satisfaction of the user when he is interacting with the system." (Daniel Roggen)

Harvest ingenuity. ".. a new research approach in pervasive computing centering on the investigation and development of human machines systems that increase the freedom, utilize the power of communities, harvest the ingenuity of a large number of independent developers, and develops technologies that address people's basic needs..." (Albrecht Schmidt)

Uncertainty. "Context-awareness is woefully limited in our computing devices and they rarely do the "right thing" or what we would prefer. We need to be able to teach them how we want them to work for us... What we need are the ability to tell if a user is interruptable, what information they likely to need next, what work/play they might be engaged in, and who might be engaged in it with them ...our models will never cover all possible activities in which humans may engage." (Gaetano Borriello)

Thinking. "Building on recent fMRI discoveries of common spatial patterns among subjects when thinking of the same word, there are numerous projects processing brain signals in an attempt to understand what people are thinking." (Daniel P. Siewiorek)

Cognitive adaptation. "One of the next grand challenges for adaptive pervasive computing will be to make devices that truly understand our mental and emotional situation and try to accommodate us." (Jennifer Healey)

Knowledge accelerators. "We need to create a techno-socio-economic knowledge accelerator - a large scale multidisciplinary project that uses current and future ICT 
developments to address the challenges of humanity involving natural scientists and engineers." (Dirk Helbing, Steven Bishop and Paul Lukowicz)

Knowledge self-organisation. "... devising the most effective mechanisms for knowledge self-organisation - including knowledge creation, propagation and dissipation - to be used both at the individual (in the workspace) and the global (in the information infrastructure) levels ..." (Andrea Omicini)

Life patterns. "...technologies allow us - for the first time in history - to collect large scale quantitative information about another fundamental realm of nature: the daily life and daily behavior of people.... One of the most interesting applications of this research considers the "quality of life" and the "life style" ...to contribute the creation of "stress-free societies". (Marco Mamei)

Collective intelligence. The importance of emerging collective intelligence cannot be denied, as it is the fact that pervasive computing technologies will make collective intelligence so deeply embedded in our activities to make it impossible ...to distinguish about what aspects of our "intelligence" are to be attributed to us as individuals, to us as member of the world society, or to us as a organs of a continuous and worldwide ICT-Social substrate. (Franco Zambonelli)

Digital formations. " ...engineering of autonomous intelligent systems that co-exist with people in real and synthetic environments - also referred to as "digital formations " or "digital spaces"... engineered to form societies, interact and compete with other ecologies, collaborate with humans and develop their own methods of conception and social norms..." (Achilles Kameas)

Software ecosystems. "A world of highly interlinked pervasive devices, smart objects and smart environments will only emerge if we succeed in unleashing economic and commercial forces that will create a self-sustaining Pervasive Software Ecosystem that provides a playing field for commercial (and non-commercial) software developers, providers, distributors, vendors and end-users." (Gerd Kortuem)

Behaviour specification. "We envision a system design methodology that relieves the developer from ["coding adaptation"] ...one should be able to specify the desired global system behavior using appropriate high-level specification languages. The pervasive system should then be endowed with an infrastructure to develop adaptation strategies for its components such that the desired global behavior is delivered across all possible situations." (Friedemann Mattern)

Programming ensembles. "... a big challenge is how to program such populations in the large. For example, one would like to state high level "suggestions" like "reduce your energy spending", "merge two populations", "please elect a leader", "spread the information by an epidemic process", "increase the security level" etc. and, ideally, the underlying population should be able to implement these in a scalable (independent of current population size) and flexible way (e.g. choose among a variety of routing methods)." (Paul Spirakis)

Digital ecosystems. "In what we refer to as 'digital ecology' theory and practice, research aims to understand and advance the interweaving of humans and ICTs to create a world with social, physical, and cyber dimensions enabling a kind of social network in which humans are not just 'consumers' of data and computing 
applications ... they are producers, 'players,' and 'inputs' whose interactions use the 'invisible hand' of the market as they interact in complex, interdependent global-scale systems in areas such as energy production and use, and neighborhood, district, city, and regional transport." (Mihaela Ulieru)

Dependability. ". . these teams have to be self-regulating, in terms of a dynamic re-allocation of roles, tasks, priorities etc., which can be specified as part of the normative system itself. A major challenge is to define dependability, in terms of being able to meet specific organizational objectives and levels of utility, at the same time being able to withstand component-loss, network outage or overload, and/or hostile behaviour." (Jeremy Pitt)

Social values. "Respect for peoples' ability to judge for themselves and be assisted by machines where needed. Respect for peoples' desire for freedom of choice and be supported by automation and decision support where appropriate. And respect for fundamental human rights, such as privacy, security and safety. A key research area is thus how to build respect for humans' social values into the fabric of machines, to deepen our understanding of 'value sensitive design'." (Sarah Spiekermann)

Trustworthiness and privacy. "There is an obvious challenge in this personalization regarding the privacy of the collected information: who is to store all this data, for how long, where, and what is it used for?" (Mark Langheinrich)

Dual spatial reality. "Problem states can more easily be transferred from the real into the digital domain (by sensors) and the results of reasoning processes of the digital domain can directly be transferred back into the real world (by actuators). This tight connection between the digital and real world is what will lead to a Dual Spatial Reality ..." (Antonio Krueger)

Mobile augmented reality. "The challenge is better connect remote people than with a mobile phone employing context aware augmented reality. Web 2.0 technologies have added to people's ability to stay connect with colleagues, friends and family ...PC's ... do not scale down to smart phone form factors. New technologies need to be investigated to overcome these issues, but in addition take advance of the mobile nature of people..." (Bruce Thomas)

Smart material. "For pervasive systems, computing is material in two ways. First, pervasive systems must intrinsically involve computing. Second, and more subtly, the computing aspects of the system must be treated the same as any other material that affects the feel and behavior of an object.... when computing is material, products will have "smart patinas", with their wear patterns determined both physically and computationally." (Tom Martin)

Meaningful applications. "We have struggled to enable large-scale explorations of socially meaningful applications. These applications include home health, elder care, and energy and resource monitoring ..." (Shwetak Patel)

Energy awareness. "... an important issue that will have great impact on how pervasive clouds will become is that of energy consumption. The development of energy-conscious and power-aware resource allocation protocols for cloud computing systems will open up more opportunities for the deployment of more pervasive technologies..." (Albert Zomaya) 
Electronic waste. "Pervasive computing at scale via portable devices has social implications in terms of electronic waste. For example, there are 4.2 billion mobile phones in use globally, with less than 3\% typically recycled according to a study ... Current mobile phones are replaced every 18-24 months, mainly to obtain devices with better performance..." (Vinod Namboodiri)

\section{Towards ICT grounded on social principles}

Human beings are born dependent, and in constant need of support by others. When growing up, humans do not gradually become independent of others, but rather become interdependent. In the course of our lives we form many give-and-take relationships, building a healthy interdependence with family, friends, communities, society and culture. "We are, at our cores, social creatures. Affiliation is the strength that allows us to join with others to create something stronger, more adaptive, and more creative than any individual: the group" (see Belonging to the Group, B.D. Perry, 2002). As Schopenhauer implies, the desire for positive social relationships is one of the most fundamental and universal of human needs. This need has deep roots in evolutionary history and exerts a powerful impact on contemporary human psychological processes. Failure to satisfy this need can have devastating consequences for psychological well-being. People who lack positive relationships often experience loneliness, guilt, jealousy, depression, and anxiety, higher incidence of psychopathology, and reduced immune system functioning. The psychological and sociological research in this areas has observed that, given the strong need for social connection, the lack or weak perception of "social affiliation" will fertilize two possible reactions: (i) antisocial-rather than affiliative - responses to exclusion [145], and (ii) an increase in motivation to build social bonds, perhaps especially with new (and possibly more promising) social partners [102]. The process of the social affiliation of a person in a societal community could be affected by distortions and obstacles in case of individuals that live the social marginalization or are at risk, because of one or more conditions of disadvantage: personal disadvantages (e.g. physical and mental disability, psychological problems, drug/alcohol addiction, being a prisoner or an ex-offender) and social disadvantages (family in economic troubles, homeless, long-term unemployment). In these cases, individuals need an external and professional support to foster the personal social inclusion, through an individualized work directed to overcome the disadvantages factors and enhance the personal and social abilities.

In our society, social services have taken up care to support social inclusion (or reintegration), for example via personal caretakers. The personalized social assistance provided is based on instruments and data coming (i) from him/her and his/her enlarged family context (primary source), (ii) friends and peer-groups, or the neighborhood (secondary source), and (iii) data records from societal authorities (schools records, banking and insurance records, clinical records, police records, etc.). While the primary and secondary sources are very important (they help the social intermediaries to know the past and present life history of an individual and establish personal contacts), they have also serious limitations: they originate from personal perceptions and records, based on the individuals ${ }^{6}$ biological senses (visual auditory, tactile) and the ability to remember, forget and elaborate the social experiences - and thus are prone to misinterpretation. Moreover, it is important to take into account that the daily life is made of multiple factors affecting the "social affiliation" of individuals as single entities and as part of larger societal bodies and the "social inclusion" in the community. The biological senses (visual auditory, tactile) and the ability to elaborate the social experiences are certainly important to feel part of the physical and social 
environment around, but they need to be read together with another phenomenon of humans that is very important: the "social awarenes", based on the "social sense", i.e. an additional human sense that helps people to perceive the "social" aspects of the environment, allowing to sense, explore and understand the social context.

The awareness of a social sense, and the ability to focus on, understand and express without personal distortions what the social context means for the individual and how it can influence a behaviour, could be the key-factors for the decisive improvement of the individualized social inclusion interventions of disadvantaged people by social intermediaries (i.e. public and private social services providers and their practitioners, associations representative of disadvantaged groups). In this respect, new technologies and ICTs could contribute to gather direct, accurate and intelligible information on how the user experiences social relations: this innovation could have very powerful effects for the enhancement of the vulnerable people social inclusion, because it would complete the set of data and information about the user personal profile provided by the traditional sources, improving the effectiveness of the inclusion personalized intervention provided by social practitioners.

In summary, the rational underlying socio-inspired ICT research is that new technologies could contribute to gather direct, accurate and intelligible information on how the user experiences social relations. This in turn could have very powerful effects on $(i)$ the design of future ICT systems per se, $(i i)$ the interaction principles between humans and ICT, but also (iii) the enhacement of a flourishing symbiosis of society and ICT overall. Towards the identification of research issues related to the potentials of underpinning new generation ICT on social principles, a variety of terms have been introduced and studied by the scientific community. Among them are:

Social context in computing is often used as a term commonly referring to the people, groups, and organizations an individual is interacting with [136]. There are some variations of this definition which are more specific and can additionally differentiate between social versus non-social context. Pentland [118] argues about the importance of social context and describes it as the additional non-verbal information (e.g. signals from the body language, facial expression, and tone of voice, etc.) transmitted among communicating people, and which is the main determinant of a successful social interaction and engagement. An application of social context in this respect, for example, can be the finding of new contacts and the integration of remote users in conversations. There has been a lot of progress in the domain of social signal processing, which builds on this definition by Pentland and focuses on non-verbal behavior in automatically recognizing social signals and social context $[148,149]$. Groh et al. [61] define social context as "all social relations, social interactions and social situations which are directly related to or confined to small time-intervals and spaceregions around the present time or present location of a person”. Schuster et al. [136] consider social context from a pervasive perspective, where they define "pervasive social context of an individual as the set of information that arises out of direct or indirect interaction with people carrying sensor-equipped pervasive devices connected to the same social network service. It comprises the explicit links, profile information and activities of people within the social graph, the joint sensor information of the pervasive devices as well as implicit information that can be inferred by combining the two." Building on Dey's definition of context (see above) [31]), social context is any information of a social nature, including both non-verbal and verbal, transmitted among communicating people, that can be used to characterize the situation of an entity.

Social sense. The biological senses (visual auditory, tactile) and the ability to elaborate the social experiences are certainly important to feel part of the physical and 
social environment around, but they need to be read together with another phenomenon of humans that is very important: the "social awareness", based on the "social sense", i.e. an additional human sense that helps people to perceive the "social" aspects of the environment, allowing to sense, explore and understand the social context.

Social awareness. In [118] Pentland identifies one key weakness in today's technology, the fact that they are socially ignorant. He writes "Technology must account for [the fact that people are social animals], by recognizing that communication is always socially situated and that discussions are not just words but part of a larger social dialogue." Additionally, initial steps have been taken by Pentland's research group to develop three socially aware platforms that objectively measure several aspects of social context, including nonlinguistic social signals. This recognition of both the larger social dialogue and the social context of a communication is what we define as social awareness. In the literature, there is no formally accepted definition of social awareness. Nowak and Conte [] identify social awareness as "... the capacity to model ongoing social processes, structures and behavioural patterns...". We build on this and define social awareness as a property that may enable technology to automatically and objectively recognize ongoing social processes, social context, social structures and behavioural patterns. Social awareness extends context awareness by considering the social dimension as the dominant feature of interest.

\subsection{Mining for social context}

Modern ICT, as for example smartphones, have started to continuously sense movements, interactions, and potentially other clues about individuals, thus also about society as a whole. Data continuously captured by hundreds of millions of personal devices around the world, promises to reveal important behavioral clues about humans in a manner never before possible. Eagle and Pentland [40] performed the first Reality Mining data collection [36], which was named by MIT Technology Review as "one of the 10 technologies most likely to change the way we live" [109].

Research using mobile phone data has mostly focused on location-driven analysis, more specifically, using Global Positioning System (GPS) data to predict transportation modes [117,127], to predict user destinations [79], or paths [4], to discover a user's stay regions (or places of interest) [110], and to predict daily step count [138]. Other location-driven tasks have made use of Global System for Mobile Communications (GSM) data for indoor localization [115] or WiFi for large-scale localization [89]. There are several works related to activity modeling from location-driven phone sensor data. CitySense [91] is a mobile application which uses GPS and WiFi data to summarize "hotspots" of activity in a city, which can then be used to make recommendations to people regarding, for example, preferred restaurants and nightclubs [69]. Liao et al. [90] use GPS data traces to label and extract a person's activities and significant places. Their method is based on Relational Markov Networks. The BeaconPrint algorithm [65] uses both WiFi and GSM to learn the places a user goes and detect if the user returns to these places.

There has also been some previous work pertaining to modeling users and their mobile phone usage patterns. Eagle and Pentland [39] use Principle Component Analysis (PCA) to identify the main components structuring daily human behavior. The main components of human activities, which are the top eigenvectors of the PCA decomposition are termed eigenbehaviors. To define the daily life of an individual in terms of eigenbehaviors, the top eigenbehaviors will show the main routines in the life of a group of users, and the remaining eigenbehaviors describe the more 
precise, non-typical behaviors in individuals' or the group's lives. Farrahi and GaticaPerez $[44,45]$ have proposed a method to discover routines of users by modeling socio-geographic cues using topic models. This methodology of discovering Reality Mining-based behaviors was then extended to determine the similarities and differences between groups of people in a computational social science experiment. The experiments are performed on a political opinion dataset, where the authors use their approach to determine the similarities and differences in the daily routines of individuals who changed political opinions versus those that do not [99]. Further, Do and Gatica-Perez [33] recently presented an analysis of application usage in smartphones, for the purpose of user retrieval. Similarly, Verkasalo et al. [147] studied the reasons and motivation behind using applications across users and non-users.

Building on the many previous works based on the sociometer [21], which is a wearable sensing device capable of sensing the amount of face-to-face interaction, conversational time, physical proximity to other people, and physical activity levels, mobile phones have been programmed to capture non-linguistic speech attributes $[92,100]$. These non verbal speech features have been used for sound classification (for example music versus voice) and for the discovery of sound events [92]. The VibeFone application[100], uses location, proximity, and tone of voice features to infer specific aspects of peoples' social lives. The mobile application has two special modes, the Jerk-o-Meter and the Wingman3G, in which VibeFone evaluates the subject's speech and provides feedback to subjects. Experiments have been conducted on several small scale data collections to measure and predict interest in conversation, and to measure attraction in a speed-dating scenario.

Other previous works in Reality Mining address a wide range of topics as follows. Wang et al. [150] model the mobility of mobile phone users to study the spreading patterns characterizing a mobile virus outbreak. They consider both location and proximity mobile phone data. They find that Bluetooth viruses spread slowly due to human mobility; however, viruses utilizing multimedia messaging services could infect all users in hours. In [37] individual calling diversity is used to explain the economic development of cities. Eagle et al. [37] find that the diversity of individuals' relationships is strongly correlated with the economic development of communities. CenceMe [108] is a personal sensing system that enables activity sharing sensed automatically by mobile phones in a user's online social network. The sensed activities, referred to as "sensing presence", captures a users' status in terms of activities (e.g., sitting, walking), disposition (e.g., happy, sad), habits (e.g., at the gym, at work), and surroundings (e.g., noisy). These features can then be shared in popular social networking sites such as Facebook, Myspace, as well as instant messaging tools such as Skype and Pidgin. Wesolowski and Eagle [152] use mobile call logs collected over a one year period to better understand one of the largest slums, Kibera, located in Nairobi, Kenya. Additionally, individual calling diversity has been used to explain the economic development of cities in [37].

There is an increasing number of works on very large-scale data collections. The dataset used by Gonzalez et al. [58], is from a phone operator, with the drawback of containing location information only when phone communication is present. They used mobile phone data to study the trajectories of human mobility patterns, and found that human trajectories show a high degree of temporal and spatial regularity, more specifically, that individual travel patterns can "collapse" into a single spatial probability distribution showing that humans follow simple, reproducible patterns. The dataset contained 100000 users over a period of six months. In [17], phone call data has been used to study the mean collective behavior of humans at large scale, focusing on the occurrence of anomalous events. The authors also investigate patterns of calling activity at the individual level and model the individual calling patterns (time between phone calls) as heavy tailed. The most recent work 
considering a very large scale mobility dataset obtained upon phone call initiation is by Phithakkitnukoon et al. [121], where they study the correlation between weather patterns and mobile phone usage.

Some state-of-the-art data collection campaigns include the Nokia-Idiap collection [76], which contains highly multimodal data on a large-scale of heterogeneous participants, consisting of family and friends, involving over 170 participants over a year of time. The data collected by Madan et al. [98] occurs over a short duration with on the order of 70 participants, however, they target specific computational social science questions during the collection, which includes the collection of detailed questionnaires and surveys from the participants. The three main motivations are human political opinions, human obesity patterns, and human health including factors such as flu symptoms and depression.

\subsection{Exploiting social context}

One of the consequences of success of PUC is the introduction of computing applications which are based on social sciences. According to Tirri [141] pervasive communication technology together with sensor technologies is on its way to fundamentally change, beside other domains, social fabric of societies. PUC provides the infrastructure to sense the environment and equips the user to interact with it seamlessly [103]. To satisfy this vision, Weiser has already in 1999 recommended that pervasive computing solutions should also be unobtrusive and transparently integrated into social behaviour [80,151].

Sensing and interaction with the environment does not only involve infrastructure elements such as digital signs (electronic displays) [42], interactive walls $[47,50,139]$ and smart floors $[63,131]$, etc., but, to apply user-adaptive or context-aware behavior, also the users themselves $[7,78,101,116]$. Since in many cases a user (agent) is more than a digital device or entity, e. g. a human being, the inclusion of social behavior into pervasive applications is increasingly gaining importance. The collective paradigm, derived from pervasive computing, social media, social networking, social signal processing, etc., has recently become known as "pervasive social computing" [160].

Recent developments within body worn sensors $[5,43,62,155]$, and ambient intelligence $[106,143]$ provide new possibilities to contribute to sensing of physical as well as cognitive/social attributes of human being, and moreover to integrate these into pervasive applications serving a smart environment [111]. The wearable systems to sense the physical characteristics such as presence, location [51], locomotion (e.g. direction and speed of arm or leg motion) [67] and body postures (e.g. sitting, standing and activity recognition) are already well developed by using accelerometers, gyroscopes, compasses and positioning/orientation sensors $[81,95,154,156]$. The new generation of wearable systems which could - for the first time- measure the cognitive aspects (e.g. tension, happiness, excitement, etc.) [104] is gaining popularity. Examples of these sensors are EOG [16], EEG [41], and ECG sensors [129] as well as galvanic skin response (GSR) sensors or pupil diameter variation sensing [35]. Similarly the development in ambient sensors to recognize the physical as well as cognitive aspects has progressed well beyond the video and audio streams analysis and has entered into implicit interaction paradigm $[128,130]$. General purpose sensing architectures have been developed, serving multi-purpose, multi-sensor, spontaneous and opportunistic sensing missions [66, 82-84].

In many of these smart environments the ultimate beneficiary are humans incorporated as social individuals. A prerequisite for the successful application of personalized services (allowing contextualization on single person granularity) is efficient, safe, and 
unobtrusive user identification and profiling. According to Pour [125, p. 42], RFID can be good solution for pervasive identification respecting privacy/security of people, the usage of mobile phones could be, after unification of interfaces, another promising approach. Despite efficient available solutions, biometrics seems the inevitable part of the future of identification as it is unique, portable, and always with the user.

In addition to a profile which defines a user, the context also involves social relationships which can be woven deep down into a profile (e.g. family members, office colleagues, relationship status, friends, etc.) or can be formulated on the fly (e.g. passengers traveling in same train carriage, fans visiting a soccer game or drivers stucking in traffic jam). Overlying, the social relation of a person is the social behavior composed of individual preferences and the collective situation.

For example, if a user is getting out of a railway station in a hurry and pushing hard within a crowd, he may be getting late for work or there is an unusual situation e.g. a mass panic [52]. A pervasive application designed to assist such a user should be aware of (i) user location, (ii) user state of arousal, (iii) user profile and (iv) environmental situation, to provide useful assistance. Knowing the location of user and the fact that he is excited, the context in which he is operating should be extracted either through usual user activity (his profile) or through situation sensing. The application should decide itself the reason for user arousal. Reaching to former reason has been a subject of context aware computing for couple of decades now. However, sensing and recognizing a crowd phenomenon is still a novice area of research due to complexity of involved social dimension. What complicate it further are human connections. For example the same user may act differently if he is accompanied with his child and perhaps he would require a different assistance from application.

Modeling a social system starts with modeling representative individual entities constituting such a system. These entities are heterogeneous with varying character and capabilities. In a social system we cannot model these entities at variable (using structural equations) or system (using differential equations) level. As an analytical method for social systems, the agent-based modeling is rapidly gaining popularity, due to its capability of directly representing individual entities and their interactions $[56]$.

\subsection{Modeling social agents}

An agent based model provides appropriate agent level features that could define a social entity [53]. These features are: (i) Autonomy: ability to make its own decisions without a central controller, (ii) Social Ability: ability to interact with other agents, (iii) Reactivity: ability to react to a stimuli, and (iv) Proactivity: ability to pursue its goal on its own initiative. Each agent in the system may have its own version of implementing these features. Additionally, an agent based model allows multiple scales of social structures culminating naturally at a macro or societal level. None of other modeling approaches, for modeling a social system, comes as natural as agent based modeling approach. Formally, agent-based modeling (ABM) is a computational method that enables to create, analyze, and experiment with models composed of agents that interact within an environment [56]. Among the features which makes $\mathrm{ABMs}$ it an attractive choice for social modeling and simulation are:

- There can be one-to-one correspondence between real world actors and virtual agents which makes it easier and natural to design an agent and also to interpret the simulation results.

- Possible heterogeneity in agents behavior advocate the usage of ABM in social systems. 
- It is possible to represent the space in which agents are acting directly into the ABM which makes modeling easier in an integrated environment.

- Using ABM, agents can interact with each other at different granularities thus introducing the core social building blocks of communication and grouping etc.

- ABM are able to implement learning / adaptation at local as well as global scale.

- Many models implicitly assume that the individuals whom they model are rational. Herbert Simon [137], criticized this and suggested that people should be modeled as boundedly rational, i.e., as limited in their cognitive abilities and thus in the degree to which they are able to optimize their utility [71]. ABM makes it easy to create boundedly rational agents. In fact, the challenge is usually not to limit the rationality of agents but to extend their intelligence to the point where they could make decisions of the same sophistication as is commonplace among people [56].

Among the major difficulties of large scale ABM simulations, on the other hand, are: (i) agents heterogeneity, (ii) overlapping granularity of interacting entities and (iii) complex space models. In the scientific computing / computational science community a generic term for second aspect is multiscale modeling which can be narrowed down as social organization in systems addressing social phenomena.

Agents' heterogeneity. In a large scale agent based system, the agents are typically heterogeneous in nature. There is a variety of behavior for each agent in chemical, biological, economic or engineering systems. However a social system addressing the cognitive aspects of participating entities (human beings) is much more complex. The following aspects highlight the challenges involved:

- Individualism: Each individual agent can be as varied as physically and behaviorally different as the humans are.

- Decision making: The process of social decision making is not a simple one. It may involve unlimited options to explore. Even a single decision may involve complex formulations. It is not practical to formulate a complete rule set before hand. The decision making rules evolve all the time as an agent learns from previous decisions and decisions taken by others. Most decisions are not independent and depends on parameters from influencing entities.

- Behavioral adaptivity: Behavioral adaptivity can be distinguished from behavioral learning as it targets the change in behavior due to dynamics of the other entities in interaction whereas learning describes the cause-affect relation which changes the rule-based with experience.

Multiscale modeling. Interaction modality (as described at agent level) is necessarily at a single scale. Multiscale modeling is the study of systems which operate at a multiple resolutions. One of the strengths of multiscale modeling is its ability to provide and link a system's functionality at different length vs. time scales [75]. An example of such a system is discussed in [75] in the domain of chemistry. The different levels of simulations (calculations) discussed are quantum mechanics calculations (within an atom), atomistic simulation (within atoms) and coarse-grained simulations (e.g. within molecules). As highlighted in the article, a major challenge for all multiscale simulations is to transfer the knowledge gained from one resolution to another. Multiscale resolution when applied in natural systems poses a major challenge due to emergence of macroscopic behavior (which is usually a subject of interest) due to microscopic interaction. The challenge arises from explicitly modeling the microscopic behavior which can vary for each of the interacting agent (as discussed in above sub-section) making simulation computationally infeasible to do sufficiently long simulations where macroscopic behavior emerges [24].

Complexity of space and agents clustering. The concept of space has two meanings; ontological and physical. In first, we take space as a room, or a specific corridor 
connecting two rooms. The conceptual definition of space in this way guarantees a more convenient behavioral analysis granularity focusing on a conceptual basis of analysis rather than unnecessary physical (coordinates etc.) details of a space. In second, we describe space in physical domain which is necessary for agent's behavioral implementation at agent level. Many observational evidence can only relate behavior of an individual on ontological level. For example an interviewee can only relate his experiences to a contextual space, or the observations reported by an ambient device may report a flow of people through an exit. This makes it difficult to extract the physical space from contextual space. Additionally a true representation of space in modeling also derives its complexity from complexity of the environment itself. Most real environments cannot easily be represented in a true-to-scale and representative digital clone.

Considering that an agent's specification includes the spatial aspects of the environment, the decision making of an agent in a social system is absolutely dependent on group size. Within a cluster it is not necessary that all the agents would/should be communicating with each other as agent's individualism describes the desire or capabilities to communicate/interact. Based on the influences from the group and agent's own expectations and experiences, an agent can perform an action or adapt its behavior. This simple specification of a social agent can intuitively be derived from ABM specifications discussed and can be used to model any social phenomena.

\subsection{Modelling trust}

While exploring a common social attribute, Sissela Bok in her seminal work: Lying: Moral Choice in Private and Public Life [11], signifies the importance of closely related issue of trust in societies as ... trust is a social good to be protected just as much as the air we breathe or the water we drink. In Golembiewski and McConkie view, Perhaps there is no single variable which so throughly influences interpersonal and group behaviour as does trust ... [57], cemented further by Luhmann as Trust ... is a basic fact of human life [94]. Nevertheless, instead of indulging into the unlimited stretch of social, psychological, and even biological aspects of trust and related issues, we have focused on formalism of trust and related issues of cooperation and collaboration in agent-based systems; a technology designed and suited for mimicking a social interaction based system like societies of humans and devices. However, the essence of factors influencing the interaction and autonomous / distributed decision making in agent-based systems still inherits its foundation from understanding and hence borrowing the concepts from social, psychological and biological means wherever it applies.

In a socio-technical system, the basic purpose is to assist the user in decisionmaking. Since the introduction of the concept of pervasive computing, the social aspects of the society and its integration into decision making process has become absolutely necessary, but at the same time a challenging task. Whats makes it even more challenging is the variation in interaction modularities; for example, human-tohuman, human-to-device, device-to-human and device-to-device interaction. Even if we ignore the almost impossible task of "precisely" quantifying a social aspect (for the purpose of using it it the system) for the time being, the mere challenge of dealing with quantifying a social aspect for these modularities is sufficiently tough. Nevertheless, a clear progress is underway primarily due to the development of appropriate modeling techniques that not only support the interactions between devices, humans and any of its combination, but also provide mechanisms similar to the decision-making mechanisms in human societies. One of the most promising technology in this regard is agent technology or an Agent-Based System (ABS). 
What makes an ABS unique w.r.t a socio-technical system is its support for "human-like" behaviour and its capability of decision-making in a cooperative fashion. An agent's capabilities of autonomy and intelligence helps reaching to a rational decision whereas capabilities of cooperation and distributed interaction helps achieving society-level goals, where a society of agents comprises of a meaningful group of agents in a population, somehow related to each other. These capabilities help agents perform localized decision making without the knowledge of global conditions (mostly analogous to the human-decision making process). The social aspects affecting the individual attributes of an agent and hence its decision making capabilities are application specific. However, whatever the application is, the very fact that the agents are working together means that trust is a relevant issue. With this report, we endeavor to understand the affects of involving trust as a social aspect affecting the decision-making process of an agent-based socio-technical system. Towards this effort, using agent based technology, a formalism of trust is also presented and used in a specific scenario.

The main work related with trust comes from the fields of sociology, (social) psychology, economics, biology and philosophy.

(Social) psychology - Morton Deutsch Perhaps the most accepted definition of trust comes from Deutsch work from 1962 [30], which states that:

- (a) the individual is confronted with an ambiguous path, a path that can lead to an event perceived to be beneficial $\left(\mathrm{Va}^{+}\right)$or to an event perceived to be harmful $\left(\mathrm{Va}^{-}\right)$;

- (b) he perceives that the occurrence of $\mathrm{Va}^{+}$or $\mathrm{Va}^{-}$is contingent on the behaviour of another person; and

- (c) he perceives the strength of $\mathrm{Va}^{-}$to be greater than the strength of $\mathrm{Va}^{+}$.

If he chooses to take an ambiguous path with such properties, I shall say he makes a trusting choice; if he chooses not to take the path, he makes a distrustful choice.

The usage of perception suggest that trust is subjective. It means that it can vary from one individual to the other, even if observable social aspect is the same. Implicitly, within the definition, there is an "cost-benefit" analysis. In many theories related to human decision making, there is a tendency of theologists to explain the behaviour using cost-benefit (or utility) analysis, as done by Deutsch. However, in practical terms, human as well as a computing system cannot spend an unlimited time on this analysis [59]. The "intelligent" guessing is always adopted by humans due to limited time in hand or sometimes laziness. This should also be the case with ABS, as computing resources would never be enough to exhaust all possibilities. The cost-benefit analysis, whether exhaustive or guessed, is always performed in certain circumstances which determines the type of trust a person is willing or forced to adopt.

Different types of trust are possible primarily based on the circumstances and individual personality (Circumstantial Trust). Deutsch explains these types with the help of a story: The Lady or the Lion. We have extended the consequences drawn from the story to explain the types of trust wherever it is necessary. The story goes on like this: There was a princess who has a suitor. The King knew about this relation and was furious. He ordered suitor into a pit which has two exits. Behind one exit there was a hungry lion waiting for the prey. Behind the other exit, there was a beautiful lady, presented as a replacement of the princess. The suitor had to make a decision knowing the two options but not exactly; behind which exit, which of his possible fate was waiting. He was also instructed to choose an exit, otherwise he would be executed. Before he made a choice, he saw princess pointing towards one of the exits. The rest is left for the imagination of the reader. 
- Trust as despair: The very fact that the suitor would be executed if he does not proceed with an exit, explains trusting a choice (making a decision) in despair, without considering the fact whether suitor follows the instruction from princess or not.

- Trust as social conformity: The decision of suitor to follow the instruction from princess or not, presumably in addition to other factors, depends on social norm set by the society. In this case, a social norm of 'woman's gentleness' may be prevalent, which may suggest to the suitor that a woman would always save life of her lover, even if he falls into the arms of another woman.

- Trust as virtue: The above argument is also true in this case. Additionally, presuming that the princess is following the social norm or virtue pointing him towards other woman, he may follow the other exit just to save his virtue.

- Trust as masochism: The difference between choice made based on virtue and masochism is the state of disappointment which forces the suitor to face the lion.

- Trust as faith: Even knowing that the princess would be pointing towards the exit having life, the suitor may follow the other exit having a faith that he would kill the lion, and would never betray princess.

- Trust as innocence: Imagine that the suitor never knew what lies behind the exits. He would make a choice; either a wild guess (in the absence of princess instruction), or a mindless following (in the presence of princess instruction).

- Trust as Risk taking/gambling: Opposed to the name given to this category, this category represents a rational behaviour given that the suitor is not influenced by emotional and normative attributes. In this case, the suitor would weigh the potential gains of wining the lady against the potential losses of losing to lion. If he thinks that there is no way he could beat the lion, he may follow the exit leading to the lady, even if he did not want to betray the princess. And there are many more combinations which could be imagined. Another aspect of gambling can be that the suitor knows that the princess would let him die rather then letting him live with another woman; in which case he may gamble against the princess's instruction.

- Trust as impulsiveness: This is similar to the Prisoner's dilemma in which a retreat in current move may guarantee a gain in future. Even knowing that there is more risk in facing the lion, the suitor may proceed towards that exit knowing that if he beats the lion, the princess would be with him. And also knowing that if he chooses the other option, he would be killed anyway; this time by princess.

- Trust as confidence: This is a more deterministic variation of gambling. In fact, the confidence about one of the choices is determined by many factors (probably all above factors). A person chooses an option in which he is most confident of utilizing all possible cost-benefit measures. That is the reason, Deutsch chooses 'confidence' as a type of trust he would be focusing on. Considering the many dimensions a confidence level is based on, Deutsch coins the term Utility to encompass everything into a function. The more the utility of a choice is (a rational choice, not necessarily unemotional), the more confident the user is about it, thus trusting it more.

Deutsch's work is guided by the following hypotheses:

- Hypothesis 1: Given that $V a^{-}$is stronger than $\mathrm{Va}^{+}$, a trusting choice will occur if: $V a^{+} * S . P .^{+}>V a^{-} * S . P .^{-}+K$. Where S.P. ${ }^{+}$is the subjective probability of attaining $\mathrm{Va}^{+}$, and $S . P .^{-}$is the subjective probability of attaining $V a^{-}$, and $\mathrm{K}$ is the constant called "security level". A cost-benefit analysis.

- Hypothesis 2: The more remote in time the possible occurrence of $\mathrm{Va}^{-}$as compared with that of $\mathrm{Va}^{+}$, the more likely is that a trusting choice will be made. The concept of memory. 
Sociology - Niklas Luhmann Luhmann's main thesis is that trust is a means for reducing the complexity of the society [94]. Instead of utility analysis, he relates trust with risk analysis, and states: It becomes ever more typical and understandable that decisions cannot avoid risk. Indeed, Trust ... presupposes a situation of risk [93]. Realization and assessment of risk does not make life any harder. In fact, without realizing and dealing with risk, it would be impossible to live a competitive and useful life due to the fact that life is too complex and short for rational manipulations.

Sociology - Bernhard Barber In his 1983 book, Logic and Limits of Trust [8], Bernhard Barber attempts to clarify the concept of trust. He relates trust with the social relations and equate it with expectations drawn from experiences within social relationship.

Can We Trust Trust? - Diego Gambetta With the deliberations from Diego Gambetta [54], we will try to conclude the discussion about definition of "trust". In his article "Can We Trust Trust?", Gambetta states that Trust is a peculiar belief predicated not on evidence but on the lack of contrary evidence - a feature that ... makes it vulnerable to deliberate destruction. Ignoring the second half of the sentence, he extends the concept of Luhmann, trying to reduce the complexity involved in cost-benefit analysis or tracking the social relationship history. He simplifies the trust relation as being trustworthy unless we are actually confronted with evidence which goes against it. In the following, we provide a review of Gambetta thesis.

... two general reasons why - even in the absence of 'thick' trust - it may be rational to trust trust and distrust distrust... The first is that if we do not, we shall never find out: trust begins with keeping oneself open to evidence, acting as if one trusted, at least until more stable beliefs can be established on the basis of further information. The second is that trust is not a resource that is depleted through use; on the contrary, the more there is the more there is likely to be ...

... if behaviour spreads through learning and imitation, then sustained distrust can only lead to further distrust. Trust, even if always misplaced, can never do worse than that, and the expectation that it might do at least marginally better is therefore plausible. However, while the previous reasons can motivate rational individuals to trust - at least to trust trust - this reason alone cannot, for though everyone may concede it, if the risk of misplacing trust is reputed to be high, no one wants to be the first to take it. It is enough, however, to motivate the search for social arrangements that may provide incentives for people to take risks.

This explains why people tend to trust rather than distrust as a default behaviour.

\section{Laying ground for socio-inspired ICT}

Advances and the confluence of contributions in core areas of computer science - like artificial intelligence, natural language processing, computer vision, robotics, machine learning, and cognitive science - have greatly contributed to what we observe to be an ICT exhibing characteristic principles of awareness. The development of more human-like computing capabilities has been the focus of several research groups in academia. However, one distinguishing feature has not been considered in much detail up until now. Computers completely lack human social capabilities. Our human social systems play critical roles in individual cognition, intelligent, and progress. In order for computers to better understand our world, particularly in a more human-like manner, one potential mostly unexplored direction is with respect to human social capabilities. Social capacity is one additional dimension of intelligence and would lead to even more intelligent computing, better usability of existing technologies, and new 
applications and technologies. To quote Pentland, "ultimately [today's communication technologies] fail because they ignore the core problem: Computers are socially ignorant" [118].

Traditionally, human social behavior has not been fully understood due to two critical factors. Firstly, humans both as individuals and groups are complex in nature. The complexity stems from differences between individuals, as well as the multiple factors which may be relevent, such as context, environment, and even the interacting individual, group size, and or seasonal effects. Secondly, large-scale social behaviorspecific data for scientific analysis is difficult to obtain. With the emergence of new forms of large-scale human behavioral sensing techniques, such as reality mining [38, 40] and computational social science (CSS) [88], a better understanding of human social behavior at larger scales is becoming a possibility. We are at the point where "big data" can help research efforts to address previously unexplored directions in computer science, particularly in an interdisciplinary manner with the social sciences.

There is an increasingly large body of research in computer science relating to human social behavior sensing, social signal processing, social awareness, social intelligence, and socio-inspired ICT. These research topics, some of which have gained more momentum than others, are all centered around human social behavior and its detection, understanding, and simulation. A very broad range of research in psychology, sociology, economics, and physics, in addition to computer science, present research on topics which are relevent. Next we define socio-inspired ICT as the transfer or automation of human social behavior understanding to ICT systems and identify several research directions from which socio-inspired ICT could greatly benefit and build upon.

\subsection{Social signals and social architectures}

Social signals have recently been defined as a communicative or informative signal that, either directly or indirectly conveys information about social actions, social interactions, social emotions, social attitudes, and social relationships [123]. Informative signals are distinguished from communicative signals based on the intention of the signal producer. If there was no direct intention of conveying a particular social information to the receiver, the signal is considered to be informative and in contrast, the signal is communicative if the emitter produces a signal with the goal of having the receiver obtain some belief [123]. Further, what characterizes "social signals" as social is not that they convey information from one entity to another, but that their "object", the type of information they convey, is social [123].

While we agree with the definition of Poggi and D'Errico [123], we believe this definition can be stated in such a way that social actions, social interactions, social emotions, social attitudes, and social relationships need not be differentiated from actions, interactions, emotions, attitudes, and relationships. To get a better insight into what social signals are and are not, we should additionally consider the term social behavior from the perspective of the definition in physiology and sociology. Social behavior is behavior directed towards society, or taking place between, members of the same species [3]. Given the previous definition of social signals [123], this would imply a person is displaying social signals with their pet, though this would not traditionally be accepted as social behavior as it is not considered true that people socialize with their dog, for example, even if they send them many emotional displays of affection. Additionally, it is not clear how anti-social signals could be differentiated from social signals. We believe the definition of social signals should additionally consider the purpose of the exchange, which is taking the interests, intentions, or needs of others into account. 
We build on the previous definition as follows. Social signals are a communicative or informative signal that either directly or indirectly conveys information about the actions, interactions, emotions, attitudes, and relationships, and necessarily takes into account the interests, intentions, or needs of others in society, or between members of the same species. This would be in contrast to anti-social signals, which would not take the interests, intentions, or needs of others into account. By taking the intention of the signal into account we remove the need to differentiate between social interactions and interactions, and so on. Socio-inspired ICT now is the recognition or automation of aspects relating to human social behavior to ICT systems. We identify two types of Social Architectures under which socio-inspired ICT research fits, the differentiation stems from the recognition versus automation goals of the ICT system. Social Artifical Intelligence The first type seeks to 'encode' human social behavior into computing. Artificial intelligence (AI) is the intelligence of machines, and the branch of computer science that aims to create it. The methodology behind AI is the understanding of human intelligence, how it works, and transferring that knowledge to computing. Similarly, the first architecure, which we call social artificial intelligence, transfers the understanding of human social behavior to computers for varying purposes, resulting in social AI. Social AI architectures can be used for improved human-computer interaction. For example, robots with human social capabilities for improved learning and development functionality, or new advancements in gaming technologies. Imagine a network of agents which human social behavior. Massive socio-inspired agent-based models would fall into this category. Replacing agents by computers, or even cars to have a network of social computers or social cars; these social AI networks could lead to many future research directions. Social Signal Recognition The second type of architecture relating to socio-inspired ICT, under which most current research can be categorized, is that where human social signals are sensed, recognized, interpreted, and processed by computers, though they do not try to simulate this behavior. Research in this category includes computing equipped with varying forms of sensors which can automatically and subjectively detect, collect, process, and correctly infer social signals.

\subsection{Cognition and social behavior: Attention and perception}

Cognitive sciences typically de-emphasize and often exclude social and emotional factors from their studies, and have been criticized for neglecting the roles of emotions and humans as inherently social beings [2]. The affective sciences consider human emotions in full detail [122]. A complete understanding of human cognition is not necessary in order to develop socio-inspired ICT, though there are several previous works in the cognitive sciences which are very relevent and the development of more advanced and complex socio-inspired ICT will likely benefit greatly from advancements in cognitive science. As aspects of critical importance in understanding social behaviour, and with that the principles of social interaction we highlight human attention and perception. Human attention is the first source of perception, consequently als awareness towards information and other individuals. Over the last decades, attention research has succeeded in identifying several attention types as well as physiological mechanisms and neural processes and revealing its relation to memory, learning, decision making and perception. In the history of attentional research many different attentional mechanisms have been discovered and according descriptive models have been developed. Usually, these models adequately describe single or several aspects of attention, e.g. the ambiguity of single- and multi-tasking capability, whereas a general, overall theory of attention is still missing. Attention research has a tradition going back to James [68] in the late 19th century. In the first 
half of the 20th century, attention has been disregarded as being 'not measurable' and it needed the First Cognitive Revolution in the middle of the 20th century for attentional research to be presentable again.

Single channel theory (SCT). The fundamental issue of attention research at its early stages was the parallelism of attentional processing. This comes down to the question whether human brain is capable of actually processing different tasks at the same time, effectively carrying out multi-tasking or if single stimuli are selected for orienting of attentional and these tasks then are processed serially. To investigate competitive selection processes, Craik [23] and especially Broadbent [14] conducted dichotic listening experiments during which they overloaded subjects with information from different sources at the same time. As a result of his experiments, Broadbent interpreted the attentional process an information processing system, which is equipped with filter mechanisms that separate relevant from irrelevant information. He concluded that humans are only capable of orienting their attention towards a single channel at a time, hence, tasks are always processed serially. In technical terms, this approach resembles a single core processor, performing scheduling processes to ensure real-time capability.

Early vs. late selection. In the ongoing, several alternative versions of the SCT evolved usually differing in less restrictive modifications of the filter mechanisms and moment of selection. Treisman [142] proposed a more flexible filter mechanism in her Feature Integration Theory, in which disregarded stimuli are merely attenuated and not completely blocked as in Broadbent's model. Deutsch \& Deutsch [29] stated that the actual filtering happens at a late processing stage and all input stimuli are processed equivalently until entering short-term memory stage. Lavie [87] combined both approaches by connecting the moment of selection to mental workload in her Perceptual Load Theory. According to her findings, early selection is carried out in case of high workload whereas in case of low workload, selection happens at a late stage.

Capacity theory. In contrast to SCT, the Capacity Theory (CT) assumes that human attention is only limited by an overall capacity of attentional resources and which can be distributed among different tasks. Following this principle, individuals are only capable of handling a certain workload, independent of dealing with single or multiple channels. CT is based on the observation that tasks can be carried out simultaneously as long as they are sufficiently automated and do not require high mental effort. The assumption that attention is depending on the overall workload is supported by experiments from Bahrick and Shelley [6], Schneider and Shiffrin [135] and Damos [26], in which training enhanced the performance of subjects in multi-tasking applications by lowering the demand for resources with growing automation. This is supported by findings from Seligmann et al. [159] and Woollacott and ShumwayCook [157] who found that usually highly automated tasks like human gait demand significant resources in case of gait disturbances. Hence, attention resources are regarded as constant and limited but freely distributable. In contrast, Kahneman [70] proposed a dynamic amount of capacity, depending on the amount of current arousal. He identifies Mental Effort as the major control component of resource allocation, being directly proportional to arousal and hence for mental resource management. This implies, that for complicated and important tasks more resources are allocated automatically, but the voluntary control over arousal and effort is severely limited. Sarter [133] describes the link between effort and arousal as the 'motivated activation of attentional systems' and developed a neuronal model which successfully connects motivation processes to neuronal activations. Still, attentional effort remains a rather neglected aspect of attention research. 
Multiple resource theory. SCT and CT cover different aspects of competitive selection depending on the task and general situation of the subject. A more general attention model has evolved in the last decades which succeeds in integrating these different attentional aspects into a single model. Wickens [153] elaborated the approach of Automaticity to a more general model of Multiple Resource Theory in which tasks can be carried out simultaneously as long as they differ in their type of resource demand. He proposes a four dimensional resource model in which he distinguishes between perceptual modalities, processing stages, visual channels and processing codes. This implies, that simultaneous performance of a visual and an auditory task causes less interference of allocated resources than performing two tasks involving related missions e.g. visual search. This model includes findings from the CT and also implies Single-Channel phenomena for tasks with a similar resource allocation. The concept of Effort is considered as a factor on filtering and selection which will be addressed in the ongoing. The history of attention research has brought forth diverse approaches and models. Whereas early theories are restricted to the description of isolated attentional mechanisms, current approaches based on and refining the Multiple Resource Theory show better overall applicability. This theory best describes the complexity of the human attentional system and is furthermore suitable for application in general and technical use-cases.

Attention and awareness. Awareness of activities is generally defined as processes which can be reported either verbally or via actions $[32,124]$. The question of human awareness is closely related to the confrontations between overt and covert attention and attention control via top-down and bottom-up processes. To some degree, it investigates whether overt and covert attention are oriented at the same destination. Moreover, it covers the question whether attention processes which have been triggered unconsciously and involuntarily by external stimuli (bottom-up processes) reach the actual awareness level or stay below the consciousness threshold. Attention and Awareness are often used interchangeably, or, stated to be linked to an inseparable degree [28]. Others soften the constrictions to the assumption that there is no attention without awareness [140]. This assumption has been disproved in recent research by Lamme [86] and Koch [77] who state that the two phenomena are closely related but not necessarily connected. Already in 1973, Kahneman [70] integrated a bypass option for the stage of conscious perception. The automatic capturing of attention by exogenous cues without necessarily triggering awareness is investigated by McCormick [105]. Duque et al. [46] worked on computing neuronal measurements to differ awareness and attention by electro-physiological signals. Brown and Ryan [15] created the Mindful Attention Awareness Scale (MAAS), a psychological awareness scale to model impact of mindfulness on well-being. MacKillop and Anderson [97] and Dam et al. [146] successfully validated this MAAS scale using different methodologies.

Intentions, goals and plans. Human behavior is motivated by goals and plans, with plans referring to conscious intentions while goals can exist at both levels of consciousness. These goals and plans are equipped with a priority attribute, which indicates how targeted the fulfillment of this goal will be pursued and how easily people can be distracted. Bisley and Goldberg [10] explored the aspect of intention and its physiological representation in the parietal lobe to infer on priority creation in attention and selection. They succeeded in identifying the lateral intraparietal area working as a priority map and being controlled by top-down and bottom-up processes. Dijksterhuis et al. [32] use the term 'goals' instead of 'intention' and assume that these are major top-down components that drive attention. Both of them are not necessarily connected to awareness. They state that, 'goals guide behavior through attention, and this guidance can occur outside of a person's awareness'. The fact that goals can be imposed unconsciously is supported by experiments of Bongers et al. [12], additionally indicating a negative influence on perceived self-esteem in 
case of failure, even on unconsciously activated goals. Okuda et al. [113] discovered an automatic regulation of attention between task performance and future goals without any intervention of conscious control systems, indicating how even task management can be carried out automatically at an unconscious level.

Emotions and Instincts. Emotions and instincts represent abstract forms of buildin goals and plans which are only directed at unconscious and automated processing. Bradley [13] concentrated on the most fundamental motivational system which is survival instinct, and identified mechanisms that affect orientation of attention and behavior. Similar to Bradley, Ohman [112] investigated how strong emotions influence automatic attention capture, and found that evolutionary relevant threatening stimuli automatically triggered fear, which supported higher arousal states and higher priorities in the selection process. In her extensive review about the effects of emotion on attention, Yiend [158] states, that there is no general pop-out effect of negative information, but the visual search for negative or threatening information runs much faster. This supports assumptions from capacity-based attention models in which additional resources can be allocated in case of states of high arousal. Phelps et al. [119] found evidence that emotion facilitates early vision, and vision is improved in the presence of emotional stimuli. Dolan [34] describes how emotion influences decisionmaking processes by relating emotions from past decisions to future determinations, thus also connecting learned experiences to future decision making and behavior.

Perception. In order to receive information from the environment the sensory organs such as the eyes, ears, and nose, receive inputs and transmits information to the brain as part of a sensory system [107]. A theoretical issue on which psychologists are divided is the extent to which perception relies directly on the information present in the stimulus. Some argue that perceptual processes are not direct, but depend on the perceiver's expectations and previous knowledge as well as the information available in the stimulus itself [107]. Two proposed processes in perception have theorized about this issue, the first is a direct theory of perception which is a 'bottom-up' theory [55] and the second is a constructivist (indirect) theory of perception and is a 'top-down' theory [60]. Bottom-up processing [55] is also known as data-driven processing, because perception begins with the stimulus itself. Processing is carried out in one direction from the retina to the visual cortex, with each successive stage in the visual pathway carrying out ever more complex analysis of the input [107]. Topdown processing [60] refers to the use of contextual information in pattern recognition. For example, understanding difficult handwriting is easier when reading complete sentences than when reading single and isolated words. This is because the meaning of the surrounding words provide a context to aid understanding [107].

From an ICT perspective, machine perception had previously been proposed as a promising future technology from a human-computer interaction perspective $[25,144]$. Along the same lines, perceptual user interfaces (PUIs) are designed to make humancomputer interaction more like how people interact with each other in the real world [144]. The chameleon effect is the nonconscious mimicry of the postures, mannerisms, facial expressions, and other behaviors of one's interaction partners, such that one's behavior passively or unintentionally changes to match that of others in one's current social environment [20]. A perception-behavior link has been suggested as the mechanism involved, whereby the mere perception of another's behavior automatically increases the likelihood of engaging in that behavior oneself [20].

\section{Concluding remarks}

An analsis of the evolution of "aware ICT" over the past two decades, together with a systematic consultation of the scientific community and a review of the literature 


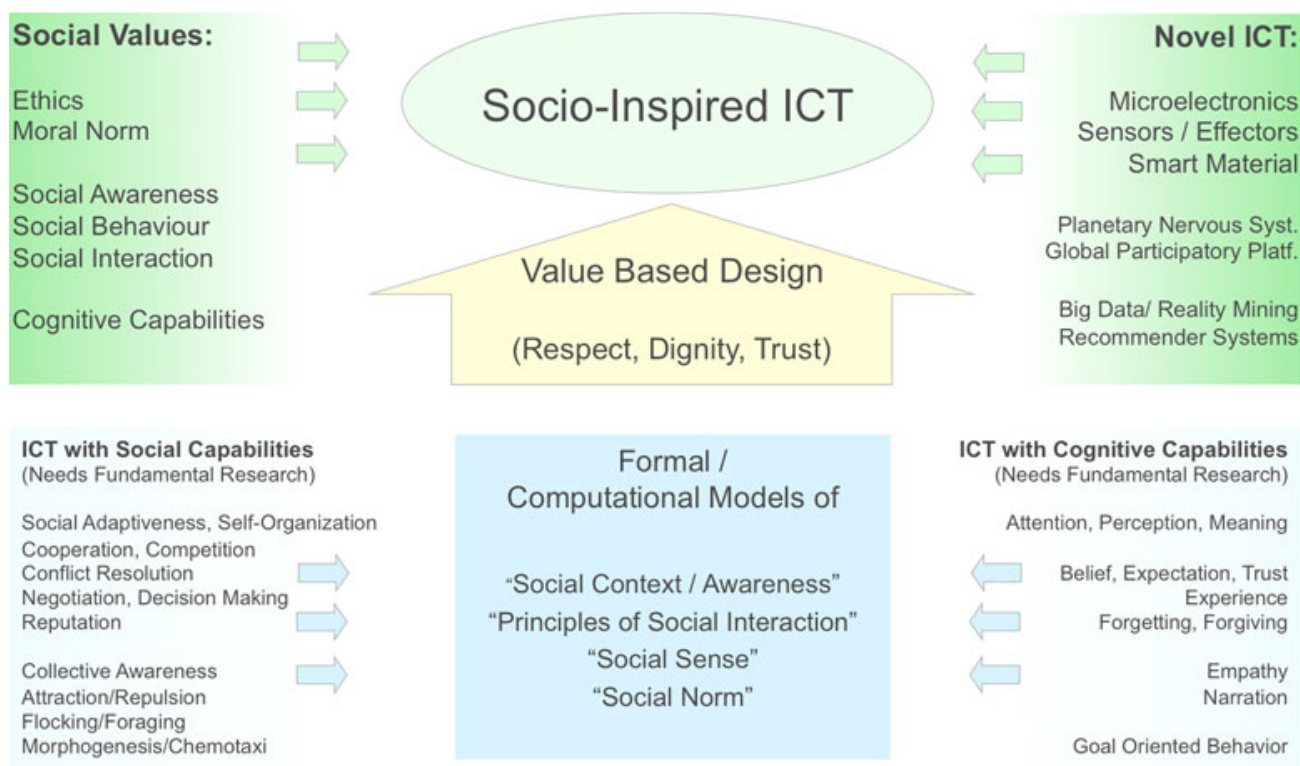

Fig. 2. Research Questions Situated around Socio-InspiredICD.

revealed needs for, features of and even first concepts towards what we call socioinspired ICT in this position paper. Current literature addresses research issues ranging from situation, to activity and even cognitive state (e.g. emotion), identifying needs to also address social capacities and bahaviours of individuals when designing, developing and deploying novel ICT.

In order to lay ground for the evolution future ICT systems, the understanding of principles of social engagement, experiencing and behavioral consequences, as well as the respect for individual and social values ("value based design", i.e. ICT designs respecting values like trust, souveregnity, respect, dignitiy, privacy, etc., see Fig. 2) reveal as an apparent, prevalent claim throughout many disciplines potentially fertilizing and steering this evolution (Computer Science, Social and Cognitive Sciences, Complexity Science). A "framework of principles" for socially adaptive ICTs is in demand, formalizing the process on how individuals engage in social activities and experience social relations, and make it the design-, implementation-, and operational principles of forthcoming large-scale ICT systems. Towards formal and computational models underpinning socio-inspired ICT, multi-scale (in time and space) sensing system able to capture, analyse and store social engagements, impressions, activities and behaviors are needed. Concepts like "social context", "social awareness" and "social sense" need to be studied and formalized both at the level od individual social beings, as well as at the level of groups, collectives, and even whole societies. Towards this, the potentials of sensor systems needed and available to capture impressions of social interactions have to be investigated, structuring sensor technology with respect to physical constraints (size, weight, etc.), mobility, energy consumptions, availability and fault tolerance, quality of service, accessibility and seamless integration. The methodological and algorithmic integration of the foundational principles of human social capabilities as well as human cognitive capabilities will ultimately allow for design-, development- and operational principles of novel ICT with social capabilities as well as cognitive capabilities. This "alignment" of future generation ICTs with the capacities and capabilities of individuals and societies gives rise for a flourishing 
symbiosis among society and ICT at the confluence of social values and technological progress.

The provision of a formal, computational framework of consolidated principles and models of $(i)$ individual socio-cognitive capacities and $(i i)$ collective social capacities appears to be the critcal precondition for a successful development of a reference architectures of globe spanning, participative, trustworthy, engaging, socially adaptive ICTs that are $(i)$ value sensitive by design, that $(i i)$ adapt to and co-evolve with the dynamics of the norms and values of societies, and (iii) center on a long-term stability of humankind.

\section{References}

1. The economist (http://www.economist.com/blogs/babbage/2011/06/virtualcurrency) (2011)

2. Stanford encyclopedia of philosophy

3. Wikipedia (http://en.wikipedia.org/wiki/Social_behavior)

4. S. Akoush, A. Sameh, Mobile user movement prediction using bayesian learning for neural networks. In: International Wireless Communications and Mobile Computing Conference (ACM IWCMC) (Honolulu, Hawaii, USA, 2007), p. 191

5. O. Amft, H. Junker, P. Lukowicz, G. Troster, C. Schuster, Sensing muscle activities with body-worn sensors. In: Wearable and Implantable Body Sensor Networks, 2006. BSN 2006. International Workshop on (2006), p. 4, doi: 10.1109/BSN.2006.48

6. H.P. Bahrick, C. Shelly, J. Exper. Psychol. 56, 288 (1958)

7. M. Baldauf, S. Dustdar, F. Rosenberg, Int. J. Ad Hoc Ubiquitous Comput. 2, 263 (2007) (http://portal.acm.org/citation.cfm?id=1356236.1356243)

8. B. Barber, Logic and Limits of Trust (Rutgers University Press, New Brunswick, N.J., 1983)

9. M. Barry, J. Gutknecht, I. Kulka, P. Lukowicz, T. Stricker, J. Mobile Multimedia 1, $112(2005)$

10. J.W. Bisley, M.E. Goldberg, Ann. Rev. Neurosci. 33, 1 (2010) (http://dx.doi.org/ 10.1146/annurev-neuro-060909-152823)

11. S. Bok, Lying: Moral Choice in Public and Private Life (New York: Vintage Books, 1999)

12. K.C. Bongers, A. Dijksterhuis, R. pears, J. Exper. Social Psychology 45, 468 (2009) (http://www.sciencedirect.com/science/article/pii/S0022103109000031)

13. M.M. Bradley, Psychophysiology 46, 1 (2009) (http://dx.doi.org/10.1111/j.14698986.2008.00702.x)

14. D.E. Broadbent, Perception and communication, 3 edn. (Pergamon Press, Oxford, 1969)

15. K.W. Brown, R.M. Ryan, J. Personality Social Psychol. 84, 822 (2003)

16. A. Bulling, D. Roggen, G. Troester, Wearable eog goggles: eye-based interaction in everyday environments. In: Proceedings of the 27th international conference extended abstracts on Human factors in computing systems, CHI '09, (ACM, New York, NY, USA, 2009), p. 3259 (http://doi.acm.org/10.1145/1520340.1520468)

17. J. Candia, M. Gonzalez, P. Wang, T. Schoenharl, G. Madey, A.L. Barabasi, J. Phys. A: Math. Theor. 41, 224 (2008)

18. R. Carvalho, L. Buzna, W. Just, D. Helbing, D. Arrowsmith, Phys. Rev., E Stat. Nonlin. Soft Matter Phys. 85, 046 (2012)

19. B. Castellani, F. Hafferty, Sociology and complexity science: a new field of inquiry (Springer Verlag, 2009)

20. T.L. Chartrand, J.A. Bargh, J. Personality Social Psychol. 76, 893 (1999)

21. T. Choudhury, A. Pentland, Sensing and modeling human networks using the sociometer. In: IEEE International Symposium on Wearable Computers (ISWC) (Washington, USA, 2003), p. 216 
22. B. Cohen, Incentives build robustness in BitTorrent (2003) (http://bitconjurer. org/BitTorrent/bittorrentecon.pdf)

23. K.J.W. Craik, British J. Psychol. General Sect. 38, 56 (1947)

24. D. Crommelin, J. Frank, ERCIM News 81: Special theme, S, 28 (2010)

25. J.L. Crowley, J. Coutaz, F. Berard, F. Bérard, Comm. ACM 43, 54 (2000)

26. D.L. Damos, Residual attention as a predictor of pilot performance. Human Factors 20, 435 (1978)

27. R.K. Dash, N.R. Jennings, D.C. Parkes, IEEE Intell. Syst. 18, 40 (2003) (http://dx.doi.org/10.1109/MIS.2003.1249168)

28. F. De Brigard, J. Prinz, Wiley Interdisciplinary Rev. Cognitive Sci. 1, 51 (2010) (http://dx.doi.org/10.1002/wcs.27)

29. J. Deutsch, D. Deutsch, Psychological Rev. 70, 51 (1963) (http://www . sciencedirect.com/science/article/B6X04-4NVH9M1-4/2/69ab54d895b71908f ceb22 af $1 \mathrm{c} 3 \mathrm{e} 335 \mathrm{e})$

30. M. Deutsch, Nebraska Symposium on Motivation., chap. Cooperation and Trust: Some Theoretical Notes (Nebraska University Press, 1962)

31. A.K. Dey, Personal Ubiquitous Comput. 5, 4 (2001) (http://dx.doi.org/10. $1007 / \mathrm{s} 007790170019)$

32. A. Dijksterhuis, H. Aarts, Ann. Rev. Psychol. 61 (2010) (http://dx.doi. org/10.1146/annurev.psych.093008.100445)

33. T. Do, D. Gatica-Perez, By their apps you shall understand them: mining largescale patterns of mobile phone usage. In: 9th International Conference on Mobile and Ubiquitous Multimedia (MUM) (Limassol, Cyprus, 2010)

34. R.J. Dolan, Science 298, 1191 (2002)

35. A. Dufresne, F. Courtemanche, S. Prom Tep, S. Senecal, Physiological measures, eye tracking and task analysis to track user reactions in user generated content. In: 7th Internationcal Conference on Methods and Techniques in Behavioral Research (Measuring Behavior, 2010), p. 218

36. N. Eagle, Machine perception and learning of complex social systems. Ph.D. thesis, Massachusetts Institute of Technology, 2005

37. N. Eagle, M. Macy, R. Claxton, Science 328, 1029 (2010)

38. N. Eagle, A. Pentland (http://reality.media.mit.edu. Reality Mining website)

39. N. Eagle, A. Pentland, Behavioral Ecol. Sociobiol. 63, 1057 (2009)

40. N. Eagle, A. Pentland, D. Lazer, Proc. National Acad. Sci. (PNAS) 106, 274 (2009)

41. D. Erdogmus, A. Adami, M. Pavel, T. Lan, S. Mathan, S. Whitlow, M. Dorneich, Cognitive state estimation based on eeg for augmented cognition. In: Neural Engineering, 2005. Conference Proceedings. 2nd International IEEE EMBS Conference on (2005), p. 566 (http://dx.doi.org/10.1109/MIS.2003.1249168)

42. J. Exeler, J. Mueller, M. Buzeck, A. Krueger, Reflectivesigns: Digital signs that adapt to audience attention. In: Pervasive 2009, International Conference on Pervasive Computing (Pervasive-09), Nara, Japan, vol. 5538/2009 (Springer Berlin/Heidelberg, 2009), p. 17 (http://dx.doi.org/10.1002/wcs.27)

43. K. Farrahi, R. Emonet, A. Ferscha, Socio-technical network analysis from wearable interactions. In: Wearable Computers (ISWC), 2012 16th International Symposium on (IEEE, 2012), p. 9

44. K. Farrahi, D. Gatica-Perez, IEEE J. Selected Topics Signal Proc. (J-STSP) 4, 746 (2010)

45. K. Farrahi, D. Gatica-Perez, ACM Transactions on Intelligent Systems and Technology, Special Issue on Intelligent Systems for Activity Recognition 2, 3:1 (2011)

46. D. Fernandez-Duque, G. Grossi, I.M. Thornton, H.J. Neville, J. Cognitive Neuroscience 15, 491 (2003) (http://dl.acm.org/citation.cfm?id=1162383.1162384)

47. A. Ferscha, From individual to collective attention-models and dynamics

48. A. Ferscha, 20 years past weiser: What's next? Pervasive Computing, IEEE 11, 52 (2012)

49. A. Ferscha, N. Davies, A. Schmidt, N. Streitz, Procedia Computer Science 7, 88 (2011) 
50. A. Ferscha, S. Vogl, Pervasive web access via public communication walls. In: Proceedings of the 1st International Conference on Pervasive Computing (Pervasive 2002), vol. 2414 (Springer LNCS, Zurich, Switzerland, 2002), p. 84 (http://www.springerlink.com/content/3v9gu49ud35euu0d/)

51. A. Ferscha, K. Zia, Lifebelt: Silent directional guidance for crowd evacuation. In: Wearable Computers, 2009. ISWC'09. International Symposium on (IEEE, 2009), p. 19

52. A. Ferscha, K. Zia, Pervasive Computing, IEEE 9, 33 (2010)

53. A. Ferscha, K. Zia, A. Riener, A. Sharpanskykh, Procedia Comp. Sci. 7, 235 (2011)

54. D. Gambetta, Trust: Making and Breaking Cooperative Relations, chap. Can We Trust Trust? (Department of Sociology, University of Oxford, 2000), p. 213

55. J.J. Gibson, The Senses Considered as Perceptual Systems (Houghton Mifflin Company 1966) (http://www.amazon.com/Senses-Considered-Perceptual-Systems/dp/ $0313239614)$

56. G.N. Gilbert, Agent-based models. Sage Publications, Inc. (2008)

57. R. Golembiewski, M. McConkie, Theories of group process., chap. The centrality of interpersonal trust in group process (New York: Wiley, 1988)

58. M.C. Gonzalez, C.A. Hidalgo, A.L. Barabasi, Nature 453, 779 (2008)

59. D. Good, Trust: Making and Breaking Cooperative Relations., chap. Individuals, Interpersonal Relations, and Trust. (Department of Sociology, University of Oxford, 2000), p. 31 (http://www.sociology.ox.ac.uk/papers/good31-48.doc)

60. R.L. Gregory, The intelligent eye (1970)

61. G. Groh, E. Lehmann, T. Wang, S. Huber, F. Hammerl, Applications for social situation models. In: Proceeding of the IADIS International Conference on Wireless Applications and Computing (2010)

62. M. Haslgruebler, C. Holzmann, DarSens: A Framework for Distributed Activity Recognition from Body-Worn Sensors. In: Proceedings of the Fifth International Conference on Body Area Networks (BodyNets'10) (Corfu Island, Greece, 2010)

63. S. Helal, W. Mann, H. El-Zabadani, J. King, Y. Kaddoura, E. Jansen, Computer 38, $50(2005)$

64. D. Helbing, Social Self-Organization: Agent-Based Simulations and Experiments to Study Emergent Social Behavior, Understanding Complex Systems (Springer 2012)

65. J. Hightower, S. Consolvo, A. Lamarca, I. Smith, J. Hughes, Learning and recognizing the places we go. In: Ubiquitous Computing (UbiComp), Tokyo, Japan (2005), p. 159

66. G. Hoelzl, M. Kurz, A. Ferscha, Goal oriented opportunistic recognition of high-level composed activities using dynamically configured hidden markov models. Procedia Computer Science 10, 308 (2012)

67. G. Hoelzl, M. Kurz, P. Halbmayer, J. Erhart, M. Matscheko, A. Ferscha, S. Eisl, J. Kaltenleithner, Locomotion@ location: When the rubber hits the road. The 9th International Conference on Autonomic Computing (ICAC2012), San Jose, California, USA (2012)

68. W. James, The Principles of Psychology, vol. 1 (Dover Publications, 1950) (http://www.worldcat.org/isbn/0486203816)

69. T. Jebara (http://www.sensenetworks.com.Sense Networks) (2008)

70. D. Kahneman, Attention and effort (Prentice-Hall, Englewood Cliffs, N.J., 1973)

71. D. Kahneman, Amer. Econom. Rev. 93, 1449 (2003)

72. J.O. Kephart, D.M. Chess, The vision of autonomic computing. Computer 36, 41 (2003)

73. A. Kesting, M. Treiber, D. Helbing, IEEE Trans. Intell. Transportation Syst. 11, 172 (2010)

74. A. Kesting, M. Treiber, M. Schonhof, D. Helbing, Transportation Research Part C: Emerging Technologies 16, 668 (2008) (http://dx.doi.org/10.1016/ j.trc.2007.12.004)

75. K.N. Kirschner, A. Arnold, A. Maass, ERCIM News 81: Special theme, S, 22 (2010)

76. N. Kiukkonen, J. Blom, O. Dousse, D. Gatica-Perez, J. Laurila, Towards rich mobile phone datasets: Lausanne data collection campaign. In: Proceedings ACM International Conference on Pervasive Services (ICPS), (Berlin, Germany, 2010) 
77. C. Koch, N. Tsuchiya, Trends Cognitive Sci. 16, 103 (2012)

78. O. Krejcar, Using of ubiquitous computing principles to develop a mobile user adaptive system framework. In: Roedunet International Conference (RoEduNet), 2010 9th (2010), p. 352

79. J. Krumm, E. Horvitz, Predestination: Inferring destinations from partial trajectories. In: Ubiquitous Computing (UbiComp), (California, USA, 2006)

80. M. Kumar, S. Das, Pervasive computing: Enabling technologies and challenges, edited by A. Zomaya, Handbook of Nature-Inspired and Innovative Computing (Springer, US, 2006), p. 613

81. K. Kunze, G. Bahle, P. Lukowicz, K. Partridge, Can magnetic field sensors replace gyroscopes in wearable sensing applications? In: Wearable Computers (ISWC), 2010 International Symposium on (2010), p. 1

82. M. Kurz, G. Hölzl, A. Ferscha, Dynamic adaptation of opportunistic sensor configurations for continuous and accurate activity recognition. In: ADAPTIVE 2012, The Fourth International Conference on Adaptive and Self-Adaptive Systems and Applications (2012), p. 13

83. M. Kurz, G. Hölzl, A. Ferscha, A. Calatroni, D. Roggen, G. Tröster, Real-time transfer and evaluation of activity recognition capabilities in an opportunistic system. In: ADAPTIVE 2011, The Third International Conference on Adaptive and Self-Adaptive Systems and Applications (2011), p. 73

84. M. Kurz, G. Hölzl, A. Ferscha, A. Calatroni, D. Roggen, G. Tröster, H. Sagha, R. Chavarriaga, J. Millán, D. Bannach, et al., International Journal of Sensors, Wireless Communications and Control, Special Issue on Autonomic and Opportunistic Communications 1 (2011)

85. S. Laemmer, D. Helbing, Self-control of traffic lights and vehicle flows in urban road networks. 0802.0403 (2008) (http://arxiv.org/abs/0802.0403)

86. V. Lamme, Neural Networks 17, 861 (2004)

87. N. Lavie, A. Hirst, J.W.d. Fockert, E. Viding, J. Exper. Psychol. General 133, 339 (2004)

88. D. Lazer, A. Pentland, L. Adamic, S. Aral, A. Barabasi, D. Brewer, N. Christakis, N. Contractor, J. Fowler, M. Gutmann, T. Jebara, G. King, M. Macy, D. Roy, M. Van Alstyne, Science 323, 721 (2009)

89. J. Letchner, D. Fox, A. LaMarca, Large-scale localization from wireless signal strength. In: National Conference on Artificial Intelligence (AAAI) (Pittsburgh, Pennsylvania, USA, 2005), p. 15

90. L. Liao, D. Fox, H. Kautz, Location-based activity recognition. In: Advances in Neural Information Processing Systems (NIPS) (Vancouver, Canada, 2006), p. 787

91. M. Loecher, T. Jebara, CitySense: Multiscale space time clustering of gps points and trajectories. In: Proceedings of the Joint Statistical Meeting (2009)

92. H. Lu, W. Pan, N.D. Lane, T. Choudhury, A.T. Campbell, Soundsense: scalable sound sensing for people-centric applications on mobile phones. In: Proc. of the 7th International Conference on Mobile Systems, Applications, and Services (MobiSys) (New York, NY, USA, 2009), p. 165

93. N. Luhmann., Trust: Making and Breaking Cooperative Relations., chap. Familiarity, Confidence, Trust: Problems and Alternatives (Department of Sociology, University of Oxford, 2000)

94. N. Luhmann, G. Poggi, Trust and power (John Wiley and Sons, 1979)

95. P. Lukowicz, O. Amft, D. Roggen, Cheng, J. Comp. 43, 92 (2010)

96. P. Lukowicz, S. Pentland, A. Ferscha, Pervasive Computing, IEEE 11, 32 (2012)

97. J. MacKillop, E.J. Anderson, J. Psychopathol. Behav. Assess. 29, 289 (2007)

98. A. Madan, M. Cebrian, S. Moturu, K. Farrahi, A. Pentland, Sensing the "health state" of our society. IEEE Pervasive Computing, Speciall Issue on Large-Scale Opportunistic Sensing (2011)

99. A. Madan, K. Farrahi, D. Gatica-Perez, A. Pentland, Pervasive sensing to model political opinions in face-to-face networks. In: Pervasive (San Francisco, USA, 2011) 
100. A. Madan, A. Pentland, Vibefones: Socially aware mobile phones. In: IEEE International Symposium on Wearable Computers (ISWC), (Montreux, Switzerland 2006)

101. A. Malatras, B. Hirsbrunner, A Context-Aware Framework to Enable Adaptation in Pervasive Computing Environments. International Conference on Network-Based Information Systems (NBiS 2009) 0, 182 (2009) (http://doi. ieeecomputersociety.org/10.1109/NBiS.2009.11)

102. Maner Jon C.N.B.R.F.S.M. DeWall, J. Personality Social Psychol. 92, 42 (2007)

103. A. Markarian, J. Favela, M. Tentori, L. Castro, Seamless interaction among heterogeneous devices in support for co-located collaboration, edited by Y. Dimitriadis, I. Zigurs, E. Gomez-Sanchez, Groupware: Design, Implementation, and Use, Lecture Notes in Computer Science, vol. 4154 (Springer Berlin, Heidelberg, 2006), p. 389

104. R. Matthews, N. McDonald, P. Hervieux, P. Turner, M. Steindorf, A wearable physiological sensor suite for unobtrusive monitoring of physiological and cognitive state. In: Engineering in Medicine and Biology Society, 2007. EMBS 2007. 29th Annual International Conference of the IEEE (IEEE, 2007), p. 5276 (http://doi.apa.org/getdoi.cfm?doi=10.1037/0096-1523.23.1.168)

105. P.A. McCormick, J. Exper. Psychol. Human Perception Perf. 23, 168 (1997) (http://doi.apa.org/getdoi.cfm?doi=10.1037/0096-1523.23.1.168)

106. D. McIlwraith, J. Pansiot, G.Z. Yang, Wearable and ambient sensor fusion for the characterisation of human motion. In: Intelligent Robots and Systems (IROS), 2010 IEEE/RSJ International Conference on (2010), p. 5505

107. S.A. McLeod, Visual perception (http://www.simplypsychology.org/perceptiontheories.html) (2007)

108. E. Miluzzo, N.D. Lane, S.B. Eisenman, A.T. Campbell, Cenceme: injecting sensing presence into social networking applications. In: Proceedings of the 2nd European conference on Smart sensing and context, EuroSSC'07 (Springer-Verlag, Berlin, Heidelberg, 2007), p. 1

109. MIT Technology Review: 10 emerging technologies 2008 (http://www . technologyreview. com/specialreports/specialreport.aspx?id=25) (2008)

110. R. Montoliu, D. Gatica-Perez, Discovering human places of interest from multimodal mobile phone data. In: 9th International Conference on Mobile and Ubiquitous Multimedia (MUM), (Limassol, Cyprus, 2010)

111. H. Nakashima, H. Aghajan, J.C. Augusto (eds.), Handbook of Ambient Intelligence and Smart Environments (Springer, 2010) ISBN: 978-0-387-93807-3

112. A. Ohman, A. Flykt, Esteves, F General 130, 466 (2001)

113. J. Okuda, S.J. Gilbert, P.W. Burgess, C.D. Frith, J.S. Simons, Neuropsychologia 49, $2258(2011)$

114. E. Ostrom, Governing the commons-The evolution of institutions for collective actions (Political economy of institutions and decisions, 1990)

115. V. Otsason, A. Varshavsky, A. Lamarca, E. de Lara, Accurate gsm indoor localization. In: Ubiquitous Computing (UbiComp) (Springer Berlin/Heidelberg, 2005), p. 141

116. C.H. Park, K.B. Sim, F. Harashima, Human adaptive system model development merged with context and emotion information. In: Industrial Electronics Society, 2004. IECON 2004. 30th Annual Conference of IEEE, vol. 1 (2004), p. 633

117. D. Patterson, L. Liao, D. Fox, H. Kautz, Inferring high-level behavior from low-level sensors. In: Ubiquitous Computing (UbiComp) (Seattle, USA, 2003), p. 73

118. A.S. Pentland, Socially aware computation and communication. Computer 38, 33 (2005) (http://dx.doi.org/10.1109/MC.2005.104)

119. E.A. Phelps, S. Ling, M. Carrasco, Psychol. Sci. 17, 292 (2006)

120. M. Philipose, K. Fishkin, M. Perkowitz, D. Patterson, D. Fox, H. Kautz, D. Hahnel, Pervasive Computing, IEEE 3, 50 (2004)

121. S. Phithakkitnukoon, T.W. Leong, Z. Smoreda, P. Olivier, PLoS ONE 7, e45,745 (2012)

122. R.W. Picard, Affective computing (MIT Press, Cambridge, MA, USA, 1997) 
123. I. Poggi, F. D'Errico, Cognitive modelling of human social signals. In: Proceedings of the 2nd international workshop on Social signal processing, SSPW '10 (2010), p. 21

124. M.I. Posner, Quarterly J. Exper. Psychol. 32, 3 (1980)

125. S.A.F. Pour, User identification roadmap towards 2020. Master thesis no. muc-2008-01, Department of Interaction and System Design, School of Engineering (Blekinge Institute of Technology, Sweden, 2008)

126. R. Rahman, T. Vinkó, D. Hales, J. Pouwelse, H. Sips, Commun. Rev. 41, 182 (2011) (http://doi.acm.org/10.1145/2043164.2018458)

127. S. Reddy, J. Burke, D. Estrin, M. Hansen, M. Strivastava, Using mobile phones to determine transportation mode. In: IEEE International Symposium on Wearable Computers (ISWC), (Pittsburgh, Pennsylvania, USA, 2008)

128. A. Riener, Continuous Authentication based on Biometrics: Data, Models, and Metrics, chap. Sitting Postures \& Electrocardiograms: A Method for Continuous and Unobtrusive Driver Authentication, IGI Global (2011), p. 30, ISBN: n.a., (in press)

129. A. Riener, M. Aly, A. Ferscha, Heart on the road: HRV analysis for monitoring a driver's affective state. In: 1st International Conference on Automotive User Interfaces and Interactive Vehicular Applications (AutomotiveUI 2009) ACM Digital Library, University of Duisburg-Essen, Essen, Germany (2009), p. 8, ISBN: 978-1-60558-571

130. A. Riener, A. Ferscha, Supporting Implicit Human-to-Vehicle Interaction: Driver Identification from Sitting Postures. In: The First Annual International Symposium on Vehicular Computing Systems (ISVCS 2008), July 22-24, 2008, Trinity College Dublin, Ireland ACM Digital Library (2008), p. 10, ISBN: 978-963-9799-27-1

131. H. Rimminen, J. Lindstroem, M. Linnavuo, R. Sepponen, Trans. Info. Tech. Biomed. 14, 1475 (2010) (http://dx.doi.org/10.1109/TITB.2010.2051956)

132. D. Roggen, A. Calatroni, K. Förster, G. Tröster, P. Lukowicz, D. Bannach, A. Ferscha, M. Kurz, G. Hölzl, H. Sagha, et al., Procedia Computer Science 7, 173 (2011)

133. M. Sarter, W.J. Gehring, R. Kozak, Brain Res. Rev. 51, 145 (2006)

134. B. Schilit, N. Adams, R. Want, Context-aware computing applications. In: Proceedings of the 1994 First Workshop on Mobile Computing Systems and Applications, WMCSA '94, IEEE Computer Society, Washington, DC, USA (1994), p. 85 (http://dx.doi.org/10.1109/WMCSA.1994.16)

135. W. Schneider, R.M. Shiffrin, Psychological Rev. 84, 1 (1977)

136. D. Schuster, A. Rosi, M. Mamei, T. Springer, M. Endler, F. Zambonelli, Pervasive social context - taxonomy and survey. ACM Transactions on Intelligent Systems and Technology (TIST) (2012)

137. H.A. Simon, Quart. J. Econom 69, 99 (1955)

138. T. Sohn, A. Varshavsky, A. Lamarca, M. Chen, T. Choudhury, I. Smith, S. Consolvo, J. Hightower, W. Griswold, E. de Lara, Mobility detection using everyday gsm traces. In: Ubiquitous Computing (UbiComp) (California, USA, 2006), p. 212

139. A. Soro, G. Paddeu, M. Lobina, Multitouch sensing for collaborative interactive walls, edited by P. Forbrig, F. Paterno, A. Pejtersen, Human-Computer Interaction Symposium, IFIP International Federation for Information Processing, vol. 272 (Springer, Boston, 2008), p. 207

140. J. Taylor, Progr. Neurobiol. 71, 305 (2003)

141. H. Tirri, Pervasive Technology that Changed the World (2010), Keynote talk at the 8th International Conference On Pervasive Computing

142. A.M. Treisman, G. Gelade, Cognitive Psychol. 12, 97 (1980)

143. J. Treur, On human aspects in ambient intelligence, edited by M. Muehlhaeuser, A. Ferscha, E. Aitenbichler, Constructing Ambient Intelligence, Communications in Computer and Information Science, vol. 11 (Springer, Berlin, Heidelberg, 2008), p. 262

144. M. Turk, Comm. ACM 43, 33 (2000)

145. J. Twenge, K. Catanese, R. Baumeister, J. Personality Social Psychol. 83, 606 (2002)

146. N.T. Van Dam, M. Earleywine, A. Borders, Personality and Individual Differences 49, 805 (2010) (http://dx.doi.org/10.1016/j.paid.2010.07.020) 
147. H. Verkasalo, C. Lopez-Nicolas, F. Molina-Castillo, H. Bouwman, Analysis of users and non-users of smartphone applications. Telematics and Informatics 27, 242 (2010)

148. A. Vinciarelli, M. Pantic, H. Bourlard, Image Vision Comput. 27, 1743 (2009)

149. A. Vinciarelli, M. Pantic, D. Heylen, C. Pelachaud, I. Poggi, F. D'Errico, Bridging the gap between social animal and unsocial machine: A survey of social signal processing. IEEE Transactions on Affective Computing (2012)

150. P. Wang, M.C. Gonzalez, C.A. Hidalgo, A.L. Barabasi, Science 324, 1071 (2009)

151. M. Weiser, Comput. Commun. Rev. 3, 3 (1999) (http://doi.acm.org/10.1145/ 329124.329126)

152. A. Wesolowski, N. Eagle, Parameterizing the dynamics of slums. In: AAAI Spring Symposium on Artificial Intelligence for Development (AI-D) (2010)

153. C.D. Wickens, J.S. McCarley, Applied attention theory (CRC Press, Boca Raton, 2008) (http://www.worldcat.org/oclc/156994621)

154. M. Wirz, D. Roggen, G. Troester, Decentralized detection of group formations from wearable acceleration sensors. In: Proceedings of the 2009 IEEE International Conference on Social Computing (IEEE Press, 2009)

155. M. Wirz, D. Roggen, G. Troester, A Methodology towards the Detection of Collective Behavior Patterns by Means of Body-Worn Sensors. In: UbiLarge workshop at the 8th International Conference on Pervasive Computing (2010), p. 4

156. M. Wirz, D. Roggen, G. Troester, A wearable, ambient sound-based approach for infrastructureless fuzzy proximity estimation. In: Proceedings of the 14th IEEE International Symposium on Wearable Computers (ISWC 2010), IEEE Computer Society (2010)

157. M. Woollacott, A. Shumway-Cook, Gait \& Posture 16, 1 (2002)

158. J. Yiend, Cognition \& Emotion 24, 3 (2010)

159. G. Yogev-Seligmann, J.M. Hausdorff, N. Giladi, Movement Disorders 23, 329 (2008) (http://dx.doi.org/10.1002/mds.21720)

160. J. Zhou, J. Sun, K. Athukorala, D. Wijekoon, Pervasive social computing: Augmenting five facets of human intelligence. In: Ubiquitous Intelligence Computing and 7th International Conference on Autonomic Trusted Computing (UIC/ATC), 2010 7th International Conference on (2010), p. 1 